\title{
Updated global analysis of neutrino oscillations in the presence of eV-scale sterile neutrinos
}

\author{
Mona Dentler, ${ }^{a}$ Álvaro Hernández-Cabezudo, ${ }^{b}$ Joachim Kopp, ${ }^{a, c}$ Pedro Machado, ${ }^{d}$ \\ Michele Maltoni, ${ }^{e}$ Ivan Martinez-Soler ${ }^{e}$ and Thomas Schwetz ${ }^{b}$ \\ ${ }^{a}$ PRISMA Cluster of Excellence, Johannes Gutenberg University Mainz, \\ Staudingerweg 9, Mainz, 55128 Germany \\ ${ }^{b}$ Institut für Kernphysik, Karlsruher Institut für Technologie (KIT), \\ Hermann-von-Helmholtz-Platz 1, Eggenstein-Leopoldshafen, 76344 Germany \\ ${ }^{c}$ Theoretical Physics Department, CERN, \\ Geneva 23, 1211 Switzerland \\ ${ }^{d}$ Theoretical Physics Department, Fermi National Accelerator Laboratory, \\ Kirk Road and Pine Street, Batavia, IL, 60510 U.S.A. \\ e Instituto de Fúsica Teórica UAM/CSIC, \\ Calle de Nicolás Cabrera 13-15, Madrid, 28049 Spain \\ E-mail: modentle@uni-mainz.de, alvaro.cabezudo@kit.edu, \\ jkopp@uni-mainz.de, pmachado@fnal.gov, michele.maltoni@csic.es, \\ ivanj.martinez@estudiante.uam.es, schwetz@kit.edu
}

ABSTRACT: We discuss the possibility to explain the anomalies in short-baseline neutrino oscillation experiments in terms of sterile neutrinos. We work in a $3+1$ framework and pay special attention to recent new data from reactor experiments, IceCube and MINOS+. We find that results from the DANSS and NEOS reactor experiments support the sterile neutrino explanation of the reactor anomaly, based on an analysis that relies solely on the relative comparison of measured reactor spectra. Global data from the $\nu_{e}$ disappearance channel favour sterile neutrino oscillations at the $3 \sigma$ level with $\Delta m_{41}^{2} \approx 1.3 \mathrm{eV}^{2}$ and $\left|U_{e 4}\right| \approx$ 0.1 , even without any assumptions on predicted reactor fluxes. In contrast, the anomalies in the $\nu_{e}$ appearance channel (dominated by LSND) are in strong tension with improved bounds on $\nu_{\mu}$ disappearance, mostly driven by MINOS+ and IceCube. Under the sterile neutrino oscillation hypothesis, the $p$-value for those data sets being consistent is less than $2.6 \times 10^{-6}$. Therefore, an explanation of the LSND anomaly in terms of sterile neutrino oscillations in the $3+1$ scenario is excluded at the $4.7 \sigma$ level. This result is robust with respect to variations in the analysis and used data, in particular it depends neither on the theoretically predicted reactor neutrino fluxes, nor on constraints from any single experiment. Irrespective of the anomalies, we provide updated constraints on the allowed mixing strengths $\left|U_{\alpha 4}\right|(\alpha=e, \mu, \tau)$ of active neutrinos with a fourth neutrino mass state in the eV range.

Keywords: Neutrino Physics, Beyond Standard Model

ArXiv EPrint: 1803.10661 


\section{Contents}

1 Introduction 1

2 Neutrino oscillations in the presence of sterile neutrinos 3

$3 \stackrel{(-)}{\nu}$ disappearance data $\quad 4$

3.1 Updated reactor analysis 5

3.2 Global $\stackrel{(-)}{\nu}$ e disappearance analysis 8

$4 \stackrel{(-)}{\nu}, \rightarrow \stackrel{(-)}{\nu}_{e}$ oscillations at short baseline $\quad 10$

$5 \stackrel{(-)}{\nu}{ }_{\mu}$ disappearance data $\quad 13$

6 Constraints on $\left|U_{\tau 4}\right| \quad 15$

$\begin{array}{lll}7 & \text { The disappearance-appearance tension } & 18\end{array}$

8 Discussion and conclusions $\quad 22$

$\begin{array}{ll}\text { A The parameter goodness-of-fit test } & 24\end{array}$

B Details of the IceCube fit $\quad 24$

\section{Introduction}

For almost two decades, the possible existence of light sterile neutrinos - new species of neutral fermions participating in neutrino oscillation - has intrigued the neutrino physics community. The excitement is fuelled in particular by a number of unexpected experimental results: an unexplained excess of electron anti-neutrinos $\left(\bar{\nu}_{e}\right)$ in a muon anti-neutrino $\left(\bar{\nu}_{\mu}\right)$ beam observed at a baseline of $\sim 30 \mathrm{~m}$ from the source in the LSND experiment [1]; a similar excess found by the MiniBooNE collaboration at higher energies and correspondingly larger baseline [2]; the disagreement between theoretically predicted $\bar{\nu}_{e}$ fluxes from nuclear reactors and observations [3, 4], known as the reactor anti-neutrino anomaly [5] (see also [6-8]); and a similar disagreement between expectations and observations in experiments using intense radioactive sources $[9,10]$.

These anomalies need to be contrasted with a large set of null results in the $\nu_{\mu} \rightarrow \nu_{\mu}$, $\nu_{e} \rightarrow \nu_{e}$, and $\nu_{\mu} \rightarrow \nu_{e}$ oscillation channels as well as the corresponding anti-neutrino channels. The observation of all of these channels overconstrains sterile neutrino models, therefore global fits of such models exhibit pronounced tension, even though different data sets on each individual oscillation channel are consistent, for recent analyses see e.g. [11-21]. 
In this work, we update our previous analyses from refs. [11, 14, 21] to incorporate new experimental results. These are in particular the following:

1. New constraints on $\bar{\nu}_{e}$ disappearance into sterile neutrinos from the reactor neutrino experiments Daya Bay [22], NEOS [23], and DANSS [24-26]. Unlike the results from previous short-baseline reactor experiments that have led to the reactor antineutrino anomaly, these new analyses are based on a comparison of measured spectra at different baselines rather than a comparison of data to theoretically predicted spectra. The new results are therefore insensitive to possible mismodelling of the $\bar{\nu}_{e}$ emission from nuclear reactors. In particular, they are insensitive to an observed, but so far unexplained, bump at neutrino energies $\sim 5 \mathrm{MeV}[27-29] .{ }^{1}$ Spectral distortions in the recent data from DANSS and NEOS lead to a hint in favour of sterile neutrinos at the $3 \sigma$ level, which supports the previous reactor anomaly independent of flux predictions.

2. Daya Bay measurements of the individual neutrino fluxes from different fissible isotopes [37]. By combining the time evolution of the observed reactor anti-neutrino spectra with the known evolution of the reactor fuel composition, the Daya Bay collaboration was able to determine independently the neutrino fluxes from the two most important fissible isotopes in a nuclear reactor, ${ }^{235} \mathrm{U}$ and ${ }^{239} \mathrm{Pu}$. Their analysis suggests that the discrepancy between predicted and observed fluxes stems mainly from ${ }^{235} \mathrm{U}$, while the neutrino flux from ${ }^{239} \mathrm{Pu}$ appears consistent with predictions. (The other potentially relevant isotopes ${ }^{238} \mathrm{U}$ and ${ }^{241} \mathrm{Pu}$ are subdominant in Daya Bay.) In contrast, oscillations into sterile neutrinos would lead to equal flux deficits in all isotopes. Implications of these results for sterile neutrino models have been discussed previously in refs. [20,21]. In our previous paper [21] we have shown that both hypotheses (free flux normalizations versus sterile neutrino oscillations) give acceptable fits to Daya Bay data, and that the preference in favour of flux rescaling decreases once Daya Bay is combined with the global reactor data. We will update those results in section 3.1 below. Finally, it has been demonstrated recently that the theoretical predictions for the time-dependence of reactor anti-neutrino fluxes on which the Daya Bay analysis is based may need to be refined [38, 39]. In particular, the present analysis accounts neither for the time-dependent equilibration of decay chains nor for the possibility of neutron capture on fission products, which would lead to a non-linear dependence of anti-neutrino fluxes on the neutron flux in the reactor [38]. Taking these effects into account, Daya Bay's preference for the flux misprediction hypothesis is estimated to drop to well below $2 \sigma$ [39].

3. Final results from OPERA [40] and ICARUS [41, 42]. Both experiments constrain sterile neutrinos mixing with electron and muon neutrinos by searching for anomalous $\nu_{\mu} \rightarrow \nu_{e}$ appearance in the CNGS beam.

\footnotetext{
${ }^{1}$ See refs. [30-35] for a discussion of possible nuclear physics or experimental origins of this bump, and ref. [36] for speculations about a possible new physics explanation.
} 
4. Searches for sterile neutrinos in MINOS/MINOS+ [43] and in $\mathrm{NO} \nu \mathrm{A}$ [44]. The first analysis combines charged current $\nu_{\mu}$ disappearance data and neutral current data from the MINOS experiment and from the MINOS+ setup operating the same detector in a higher energy beam. The second analysis is based on neutral current data from $\mathrm{NO} \nu \mathrm{A}$. Especially the MINOS/MINOS+ analysis places stringent bounds on sterile neutrino mixing with $\nu_{\mu}$ over a wide range of masses.

5. New solar neutrino data, including the 2055-day energy and day/night asymmetry spectrum from Super-Kamiokande phase 4 [45] and the measurement of neutrinos from the proton-proton $(p p)$ fusion chain in the Sun recently presented by Borexino [46]. In addition, the results of all solar experiments have been updated to match the new solar neutrino fluxes predicted by the GS98 version of the Standard Solar Model presented in ref. [47].

6. Improved atmospheric neutrino data from Super-Kamiokande (including 1775 days of phase 4 data) from ref. [48], as well as the complete set of DeepCore 3-year data presented in ref. [49] and publicly released in ref. [50]. The calculations of atmospheric neutrino event rates for both detectors are based on the atmospheric neutrino flux calculations described in ref. [51].

7. First sterile neutrino limits from IceCube, based on one year of data [52-54]. This novel analysis exploits the fact that active-to-sterile oscillations of atmospheric neutrinos inside the Earth may be enhanced by a Mikheyev-Smirnov-Wolfenstein (MSW) resonance $[55,56]$. The resonance affects the anti-neutrino sector, and for sterile neutrino masses around $1 \mathrm{eV}$ occurs at energies of order $1 \mathrm{TeV}$, an energy well above IceCube's detection threshold, but still low enough to benefit from a substantial flux $[57,58]$. Consequently, IceCube is able to set strong limits on sterile neutrino mixing with $\nu_{\mu}$.

We will begin in section 2 by reviewing the formalism of neutrino oscillations in the presence of sterile neutrinos. Along the way, we will also fix our notation, such as our parameterization of the leptonic mixing matrix. In sections 3 to 5 , we will then discuss the status of the global data sets in the $\nu_{e} \rightarrow \nu_{e}, \nu_{\mu} \rightarrow \nu_{e}$, and $\nu_{\mu} \rightarrow \nu_{\mu}$ channels (and the corresponding anti-neutrino channels) in turn. In particular, section 3 discusses the recent hints from reactor spectral data and section 4 reviews the anomalies in the appearance channel. In sections 5 and 6 , we present updated constraints on the mixing of a sterile neutrino with the $\nu_{\mu}$ and $\nu_{\tau}$ flavour from global data, respectively. We will finally combine all oscillation channels in section 7 into a global fit. We will determine the goodness of fit at the global best fit point and quantify the tension between appearance and disappearance data. We will summarize our results and conclude in section 8. Supplementary material can be found in the appendices.

\section{Neutrino oscillations in the presence of sterile neutrinos}

The topic of this paper are scenarios in which the standard three-flavor framework for neutrino oscillations is augmented by adding one sterile neutrinos $\nu_{s}$. We will refer to such 
scenarios as " $3+1$ models". We will comment on scenarios with more than one sterile neutrino in section 8 .

The oscillation probability for $\nu_{\alpha} \rightarrow \nu_{\beta}$ transitions in vacuum $(\alpha, \beta=e, \mu, \tau, s)$ is given by

$$
P_{\alpha \beta}=\sum_{j, k=1}^{4} U_{\alpha j}^{*} U_{\beta j} U_{\alpha k} U_{\beta k}^{*} \exp \left[-i \frac{\Delta m_{j k}^{2} L}{2 E}\right] .
$$

Here, $L$ is the baseline, $E$ is the neutrino energy, $U_{\alpha j}$ are the elements of the leptonic mixing matrix (which is $4 \times 4$ in a $3+1$ model), and $\Delta m_{j k}^{2} \equiv m_{j}^{2}-m_{k}^{2}$ are the mass squared differences, with $m_{j}$ the neutrino mass eigenvalues. We will assume $m_{1,2,3} \ll 1 \mathrm{eV}$, but allow $m_{4}$ to be larger, thus considering the case $\Delta m_{41}^{2}>0$. For experiments in which matter effects play a significant role, in general the evolution equation should be solved numerically. In cases where a constant matter density is a good approximation, $U_{\alpha j}$ and $\Delta m_{j k}^{2}$ in eq. (2.1) can be replaced by an effective mixing matrix and effective mass squared differences in matter. For anti-neutrino oscillations, $U$ should be replaced by $U^{*}$.

The mixing matrix $U$ in vacuum can be written as a product of two-dimensional rotation matrices. Where an explicit parameterization is required, we choose

$$
U \equiv R_{34}\left(\theta_{34}\right) R_{24}\left(\theta_{24}, \delta_{24}\right) R_{14}\left(\theta_{14}\right) R_{23}\left(\theta_{23}\right) R_{13}\left(\theta_{13}, \delta_{13}\right) R_{12}\left(\theta_{12}, \delta_{12}\right)
$$

where $R_{i j}\left(\theta_{i j}\right)$ denotes a real rotation matrix in the $(i j)$-plane with rotation angle $\theta_{i j}$, and $R_{i j}\left(\theta_{i j}, \delta_{i j}\right)$ includes in addition a complex phase $\delta_{i j}$. In most cases, however, we will present our results in terms of the parameterization-independent matrix elements $U_{\alpha \beta}$.

For the following discussion the so-called short-baseline limit of eq. (2.1) will be useful. This limit refers to the situation where $\Delta m_{21}^{2} L / 4 E \ll 1, \Delta m_{31}^{2} L / 4 E \ll 1$, so that standard three-flavor oscillations have not had time to develop yet. In this case, eq. (2.1) generically simplifies to

$$
\begin{array}{ll}
P_{\alpha \alpha}^{\mathrm{SBL}}=1-4\left|U_{\alpha 4}\right|^{2}\left(1-\left|U_{\alpha 4}\right|^{2}\right) \sin ^{2}\left(\frac{\Delta m_{41}^{2} L}{4 E}\right), & (\alpha \neq \beta) . \\
P_{\alpha \beta}^{\mathrm{SBL}}=4\left|U_{\alpha 4}\right|^{2}\left|U_{\beta 4}\right|^{2} \sin ^{2}\left(\frac{\Delta m_{41}^{2} L}{4 E}\right) \quad(\alpha \neq \beta)
\end{array}
$$

As we will see later, the connection between the $\nu_{e} \rightarrow \nu_{e}, \nu_{\mu} \rightarrow \nu_{\mu}$, and $\nu_{\mu} \rightarrow \nu_{e}$ oscillation probabilities, inferred from these equations, will prove to be crucial to test the compatibility between different oscillation data sets.

An extended discussion of various other limiting cases and the corresponding parameter dependencies (including complex phases) can be found in ref. [14].

\section{$3 \stackrel{(-)}{\nu}$ disappearance data}

In the $\nu_{e}$ and $\bar{\nu}_{e}$ disappearance channels, the most important constraints on sterile neutrinos come from reactor experiments at short baseline $(L \lesssim 1 \mathrm{~km})$. But we include also data from solar neutrinos, $\nu_{e}$ scattering on ${ }^{12} \mathrm{C}$, and radioactive source experiments. The data 


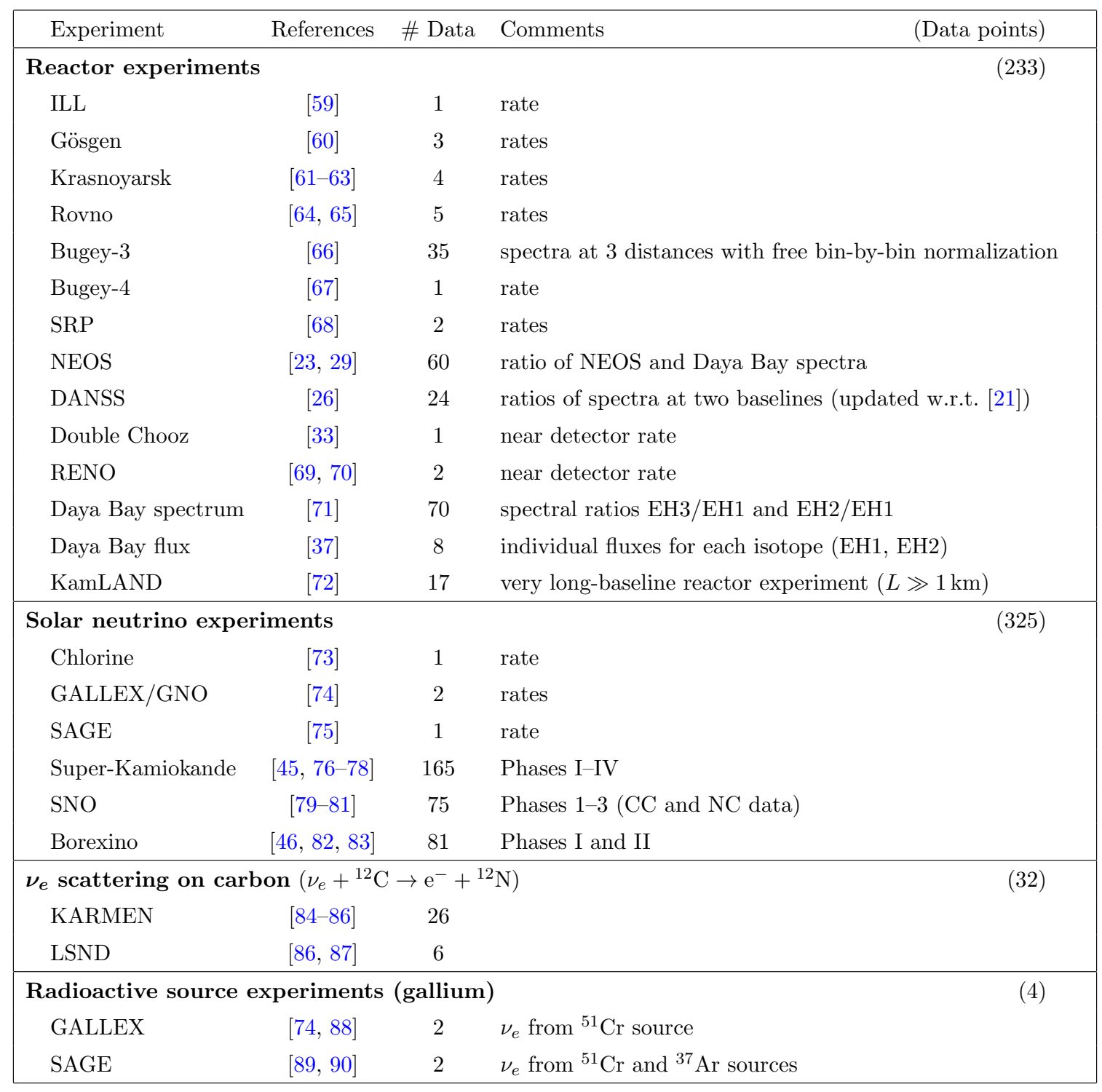

Table 1. Data sets included in our $\nu_{e} / \bar{\nu}_{e}$ disappearance analysis. The total number of data points is 594. More details can be found in ref. [21]; the only update with respect of [21] is new data from DANSS [26].

is summarized in table 1. The following analysis is based on our earlier publication [21] where more details can be found. In section 3.1 we give an update of the reactor neutrino analysis, high-lighting the impact of the recent results from the DANSS experiment [26], whereas in section 3.2 we present the global $\stackrel{(-)}{\nu})$ disappearance analysis.

\subsection{Updated reactor analysis}

The reactor analysis includes the experiments listed in table 1 . The fit by now is dominated largely by the recent NEOS [23] and DANSS [26] results, as well as the latest data from Daya Bay. For the latter we include the ratios of spectra measured in experimental halls (EH) 3 and 1, and in experimental halls 2 and 1 [71], as well as the measurement of 
the individual neutrino fluxes from each fissible isotope [37]. The analysis presented here is based largely on ref. [21] where more details can be found. The important difference with respect to that analysis is the recent preliminary results from the DANSS experiment presented in December 2017 [26], which consists of a data sample of approximately four times increased exposure compared to the one shown in March 2017 [25] used in [21]. Another recent analysis including this latest DANSS data can be found in ref. [91].

Regarding reactor neutrino flux predictions we consider two scenarios: (i) fixed fluxes, where we set the uncertainties on the predicted anti-neutrino fluxes to the values estimated in the original publications [3, 4]; (ii) free fluxes, where the normalizations of the neutrino fluxes from the four main fissible isotopes ${ }^{235} \mathrm{U},{ }^{238} \mathrm{U},{ }^{239} \mathrm{Pu}$ and ${ }^{241} \mathrm{Pu}$ are allowed to float freely. (A weak constraint $\pm 20 \%$ at $1 \sigma$ is included for the numerically subdominant fluxes from ${ }^{238} \mathrm{U}$ and ${ }^{241} \mathrm{Pu}$ to avoid unphysical values.) Note that we never rely on the predicted anti-neutrino spectra, only on the predicted rates. Even in the case of fixed fluxes, those analyses which use spectral information are based entirely on ratios of spectra at different baselines.

The new spectral data from DANSS are shown in the left panel of figure 1. The DANSS experiment uses a movable detector. The plot shows the ratio of the spectra observed in two detector locations corresponding to baselines of 10.7 and $12.7 \mathrm{~m}$. The data show a spectral distortion, leading to a preference in favour of sterile neutrino oscillations, as illustrated by the orange curve in figure 2. The remarkable observation is that the preferred region from DANSS overlaps with the one from NEOS, which also observes a spectral distortion consistent with sterile neutrino oscillations, see right panel of figure 1. Results of the combined analysis of DANSS and NEOS are given in table 2. The best fit of NEOS + DayaBay is located at $\Delta m_{41}^{2}=1.78 \mathrm{eV}^{2}$, however there is also a local minimum around $\Delta m_{41}^{2}=1.3 \mathrm{eV}^{2}$ consistent with DANSS. Note that the NEOS spectrum is statistically dominated by the low energy part, where the spectra for those two values of $\Delta m_{41}^{2}$ are similar, as shown in the right panel of figure 1. We find that the no-oscillation hypothesis is disfavoured with respect to sterile neutrino oscillations at a significance of $3.3 \sigma$. Let us stress that this result is completely independent of reactor neutrino flux predictions. It is only based on bin-by-bin spectral comparison between two detector locations in DANSS, and between the spectra observed in NEOS and Daya Bay.

Combing all available reactor data, we obtain the results shown in table 2 and figure 2 . These results confirm the $\simeq 3 \sigma$ hint in favour of sterile neutrinos from DANSS and NEOS in the analysis with free fluxes. If the fluxes are fixed and the predicted neutrino rate is used ("reactor anomaly"), the significance increases to $3.5 \sigma$, with a best fit point consistent with the DANSS/NEOS spectral indications. Note that in the analysis using fixed fluxes there is minor tension between "old" reactor data and the DANSS/NEOS best fit region, see figure 2. Despite this small tension, the significance for sterile neutrinos increases from $3.3 \sigma$ for NEOS+DANSS to $3.5 \sigma$ for the global data. We conclude that recent data support the indication in favour of sterile neutrinos from the reactor anomaly, a conclusion that is solely based on spectral distortions, but independent of reactor flux predictions.

Let us comment on the impact of the Daya Bay measurements of the individual neutrino fluxes from different fissible isotopes [37] by using the time evolution of the observed 


\begin{tabular}{|c|c|c|c|c|c|}
\hline Analysis & $\Delta m_{41}^{2}\left[\mathrm{eV}^{2}\right]$ & $\left|U_{e 4}^{2}\right|$ & $\chi_{\min }^{2} /$ dof & $\Delta \chi^{2}$ (no-osc) & significance \\
\hline DANSS+NEOS & 1.3 & 0.00964 & $74.4 /(84-2)$ & 13.6 & $3.3 \sigma$ \\
\hline all reactor (flux-free) & 1.3 & 0.00887 & $185.8 /(233-5)$ & 11.5 & $2.9 \sigma$ \\
\hline all reactor (flux-fixed) & 1.3 & 0.00964 & $196.0 /(233-3)$ & 15.5 & $3.5 \sigma$ \\
\hline$\stackrel{(-)}{\nu}_{e}$ disap. (flux-free) & 1.3 & 0.00901 & $542.9 /(594-8)$ & 13.4 & $3.2 \sigma$ \\
\hline$\stackrel{(-)}{\nu}_{e}$ disap. (flux-fixed) & 1.3 & 0.0102 & $552.8 /(594-6)$ & 17.5 & $3.8 \sigma$ \\
\hline
\end{tabular}

Table 2. Results on $\stackrel{(-)}{\nu}$ disappearance from DANSS+NEOS, from a fit to all reactor data (both for free fluxes and fixed fluxes), and from a fit to the combined $\stackrel{(-)}{\nu}$ e disappearance data listed in table 1. For each combination of data sets, we give the parameter values and the $\chi^{2}$ value per degree of freedom at the best fit point. In all fits, we treat $\theta_{14}$ and $\Delta m_{41}^{2}$ as free parameters. For the "all reactor" sample, we also leave $\theta_{13}$ free. In the " $\frac{(-)}{\nu} e_{e}$ disap." analyses, all parameters listed in eq. (3.2) are allowed to float. For the analyses with free reactor fluxes, there are two additional free parameters corresponding to the normalization of the ${ }^{235} \mathrm{U}$ and ${ }^{239} \mathrm{Pu}$ fluxes. The last two columns of the table give the $\Delta \chi^{2}$ between the no-oscillation hypothesis and the best fit, as well as the significance at which the no-oscillation hypothesis is disfavoured. It is obtained by assuming that $\Delta \chi^{2}$ follows a $\chi^{2}$ distribution with two degrees of freedom $\left(\Delta m_{41}^{2}\right.$ and $\left.\left|U_{e 4}\right|\right)$.
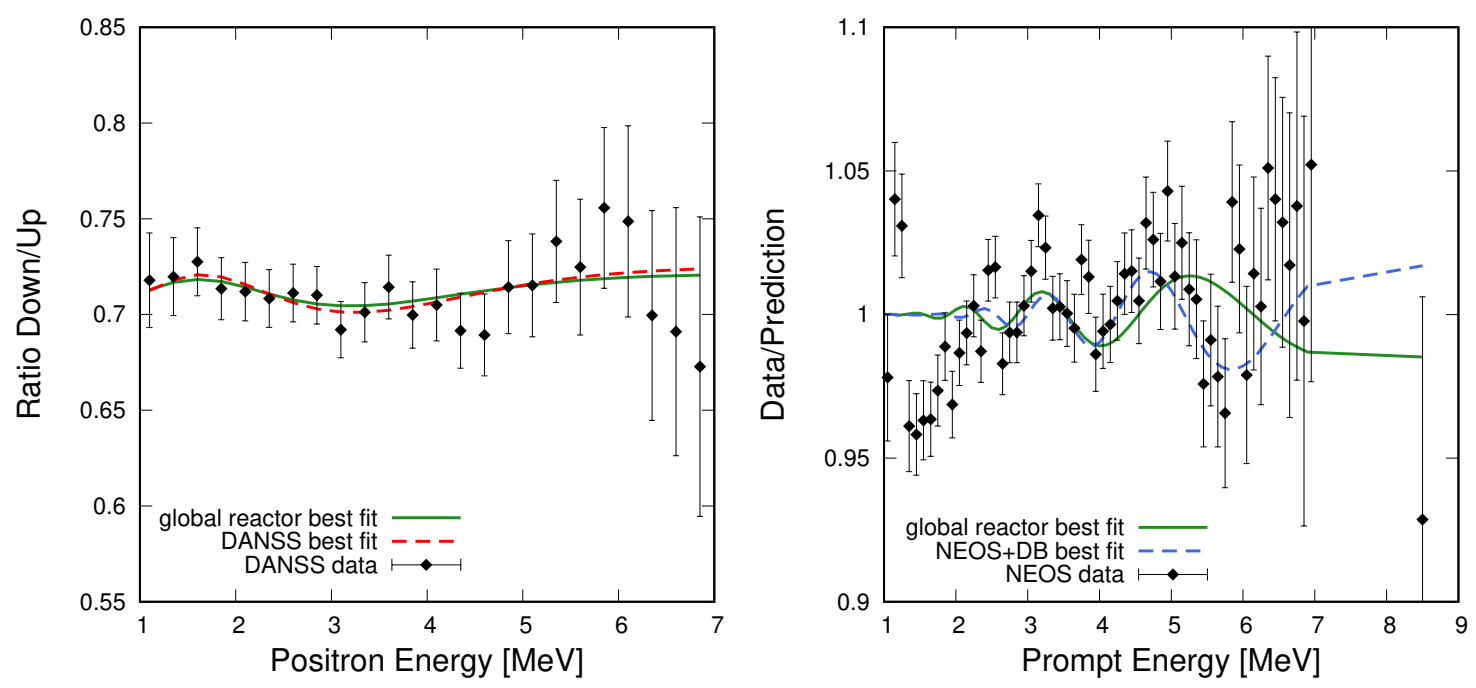

Figure 1. Observed spectra for the DANSS (left) and NEOS (right) experiments compared to the predicted spectra at the individual best fit points (dashed) and the best fit point from a global analysis of all reactor data (solid). The left panel shows the ratio of the observed event rates at the two detector locations in DANSS (24 bins). The right panel shows the NEOS spectral data relative to the prediction extrapolated from the measured Day Bay spectrum (60 bins). The best fit points are $\Delta m_{41}^{2}=1.32 \mathrm{eV}^{2}, \sin ^{2} \theta_{14}=0.012$ for DANSS, $\Delta m_{41}^{2}=1.78 \mathrm{eV}^{2}, \sin ^{2} \theta_{14}=0.013$ for NEOS + Daya Bay, and $\Delta m_{41}^{2}=1.29 \mathrm{eV}^{2}, \sin ^{2} \theta_{14}=0.0089$ for the fit to all reactor data, assuming a free normalization for the neutrino fluxes from the four main fissible isotopes. 


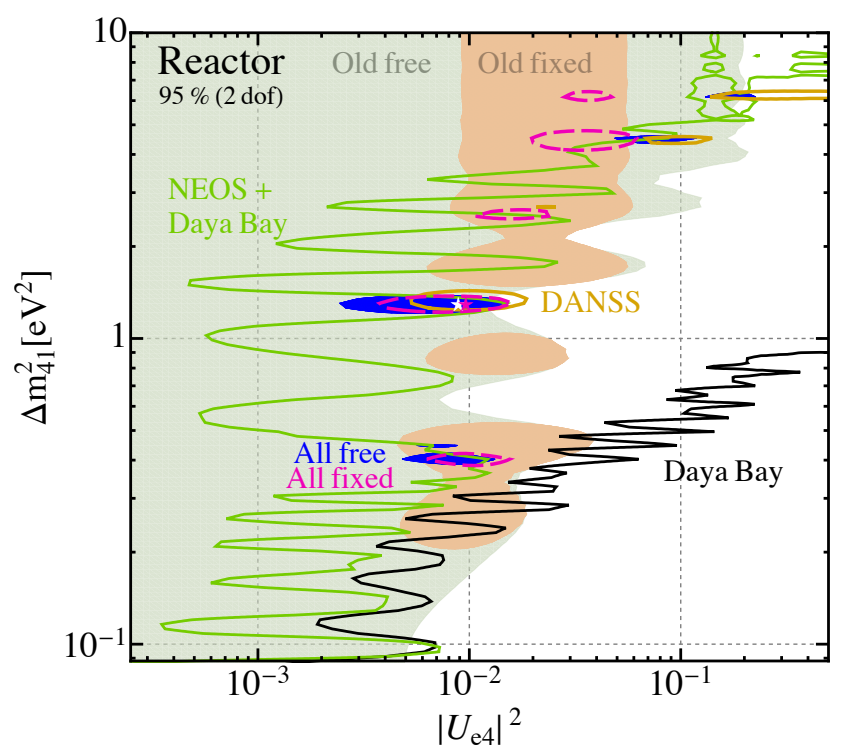

Figure 2. Allowed regions at 95\% CL (2 dof) from reactor data. The solid curves correspond to Daya Bay spectral data (black), NEOS + Daya Bay (green), and DANSS (orange); they are independent of assumptions on fluxes because they are only based on spectral ratios. The lightshaded areas labelled "old" correspond to all data from table 1 except Daya Bay, DANSS, NEOS, and they are shown for the flux-free analysis making no assumptions about flux normalization and spectra (light green), as well as for the flux-fixed analysis (light orange), assuming reactor flux predictions and their published uncertainties. The blue shaded regions correspond to all reactor data from table 1 for the flux-free analysis, whereas the dashed magenta contours indicate the global data for the flux fixed analysis. The white (pink) star indicates the best fit point $\Delta m_{41}^{2}=1.29 \mathrm{eV}^{2}$, $\sin ^{2} \theta_{14}=0.0089\left(\Delta m_{41}^{2}=1.29 \mathrm{eV}^{2}, \sin ^{2} \theta_{14}=0.0096\right)$ for free (fixed) reactor fluxes.

reactor anti-neutrino spectra. These data have been used to compare the hypothesis $H_{1}$ of no-oscillations but free flux normalizations to the hypothesis $H_{0}$ that flux predictions $[3,4]$ (including their error estimates) are correct and a sterile neutrino exists. Considering the test statistic

$$
T=\chi_{\min }^{2}\left(H_{0}\right)-\chi_{\min }^{2}\left(H_{1}\right),
$$

Daya Bay data lead to $T_{\mathrm{obs}}=6.3$, which prefers $H_{1}$ (flux-free) over $H_{0}$ (oscillations) at $2.7 \sigma[21,37]$ (see, however, [39]). As shown previously [20, 21], this preference decreases, once the global reactor data is combined with DayaBay data. Using the numbers given in table 2 , we find that with present combined reactor data, $T_{\mathrm{obs}}=-1.3$, which actually shows a slight preference for oscillations over the no-oscillation but flux-free hypothesis. Again the main driver for this are spectral distortions, which can be fit better by oscillations than by re-scaling fluxes.

\subsection{Global $\stackrel{(-)}{\nu}$ e disappearance analysis}

We proceed now to combining reactor data with all other data on $\stackrel{(-)}{\nu}_{e}$ disappearance listed in table 1. In fitting these data we scan the following set of parameters (see eq. (2.2) for 


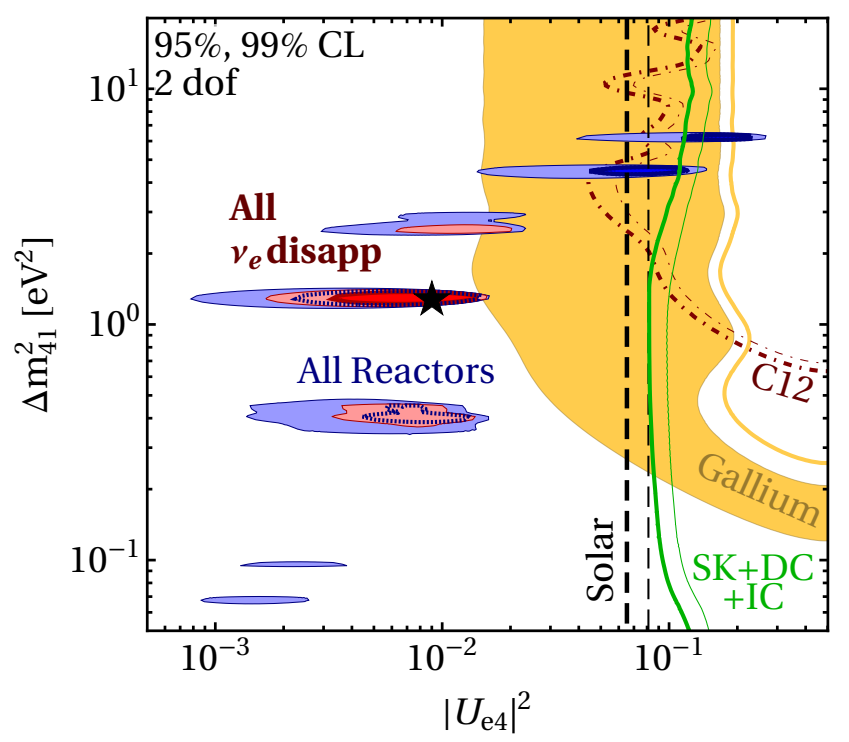

Figure 3. Constraints on $\nu_{e} / \bar{\nu}_{e}$ disappearance in the $3+1$ scenario. We show the preferred parameter regions at $95 \%$ and $99 \% \mathrm{CL}$, projected onto the plane spanned by the mixing matrix element $\left|U_{e 4}\right|^{2}$ and the mass squared difference $\Delta m_{41}^{2}$. The parameter space inside the shaded areas and to the left of the exclusion curves is allowed. For the reactor analysis we adopt the conservative assumption of free flux normalizations. The red region includes all data listed in table 1 . The green curves show the limit on $\left|U_{e 4}\right|^{2}$ obtained from atmospheric neutrino data from SuperK, IceCube and DeepCore, discussed in section 5.

our mixing matrix convention):

$$
\Delta m_{31}^{2}, \quad \Delta m_{41}^{2}, \quad \theta_{12}, \quad \theta_{14}, \quad \theta_{24}, \quad \theta_{34} .
$$

We fix $\theta_{13}$ here since it is determined very accurately, and we have checked that its best fit value does not depend on the possible existence of sterile neutrinos [14]. The dependence on $\theta_{24}$ and $\theta_{34}$ appears due to solar neutrino data, which in addition to the $\nu_{e}$ survival probability includes also NC data sensitive to $\nu_{e} \rightarrow \nu_{s}$ transitions. $^{2}$ The results are shown in the last two rows of table 2 and in figure 3 . We observe that the best fit point remains stable at $\Delta m_{41}^{2} \approx 1.3 \mathrm{eV}^{2}$, in agreement with the reactor-only analysis.

From figure 3 we observe a slight tension between the global best fit point and the region favoured by the gallium anomaly. We have used the parameter goodness-of-fit (PG) test [92] to quantify the compatibility of the gallium anomaly with reactor data. We obtain for the PG test-statistic (see appendix A for a review) $\chi_{\mathrm{PG}}^{2}=4.7$, irrespective of whether reactor fluxes are fixed or free. For 2 dof, this translates into a $p$-value of about $9 \%$ for the compatibility of reactors and gallium. From figure 3 we see, however, that the combined best fit point of reactor and gallium data lies in the island around $\Delta m_{41}^{2} \approx 4.5 \mathrm{eV}^{2}$, which is disfavoured by solar neutrinos as well as neutrino scattering on ${ }^{12} \mathrm{C}$. For the global best

\footnotetext{
${ }^{2}$ Formally solar neutrino data depend also on complex phases [14]. In our numerical scan we do take this effect into account. However, we have checked that the dependence is marginal and therefore we do not include phases in the counting of full degrees of freedom.
} 
fit point around $\Delta m_{41}^{2} \approx 1.3 \mathrm{eV}^{2}$, the $\mathrm{PG}$ test comparing reactor and gallium data gives $\chi_{\mathrm{PG}}^{2}=6.9$ (7.2) for fixed fluxes (free fluxes). This corresponds to a $p$-value of $3.1 \%(2.8 \%)$, indicating some minor tension between these data sets. Despite this tension, table 2 shows that the significance of rejecting no-oscillations of the combined fit increases by about two units in $\Delta \chi^{2}$ compared to the reactor-only analysis, both for the flux-free and flux-fixed analyses.

In figure 3 we show also the bound on $\left|U_{e 4}\right|^{2}$ obtained from the atmospheric neutrino experiments SuperKamiokande (SK), IceCube (IC), and DeepCore (DC), see section 5 for more details. We observe that this bound is comparable to the one from solar neutrino data. The effect of sterile neutrinos on low-energy atmospheric data as relevant for SK and DC has been discussed in the appendix of ref. [93]. It amounts mostly to a normalization effect of the electron and muon neutrino survival probability according to $P_{\alpha \alpha} \propto\left(1-2\left|U_{\alpha 4}\right|^{2}\right)$ with $\alpha=e, \mu$. In our SK/DC analyses we assume a $20 \%$ correlated normalization error on $e$ and $\mu$-like events, and a $5 \%$ error on the ratio of them. Therefore, we can expect a $1 \sigma$ bound of order 0.1 on $\left|U_{\alpha 4}\right|^{2}$ from those data alone. If either $\left|U_{e 4}\right|^{2}$ or $\left|U_{\mu 4}\right|^{2}$ is independently constrained from any other data, the bound on the other one from SK/DC becomes significantly stronger, due to the correlated uncertainty. Since the high-energy data relevant for IC provide such an independent constraint on $\left|U_{\mu 4}\right|^{2}$ due to the resonant matter effect (see section 5), the combined bound improves and we get $\left|U_{e 4}\right|^{2} \lesssim 0.1$ at 99\% CL (2 dof). Note that we do not include atmospheric data in the global $\stackrel{(-)}{\nu}$ e disappearance analysis presented in this section, since in this work we classify atmospheric neutrino experiments as $\stackrel{(-)}{\nu}_{\mu}$ disappearance to be discussed below.

We conclude that global $\stackrel{(-)}{\nu}$ disappearance data show a robust hint in favour of sterile neutrinos at the $3 \sigma$ level, independent of reactor flux predictions. If reactor flux predictions (including their uncertainties) are assumed to be correct, the significance reaches $3.8 \sigma$.

\section{$4 \stackrel{(-)}{\nu}, \stackrel{(-)}{\nu}_{e}$ oscillations at short baseline}

The appearance channel $\bar{\nu}_{\mu} \rightarrow \bar{\nu}_{e}$ was the first oscillation channel to reveal possible hints for sterile neutrinos, namely in the LSND experiment [1]. This hint, which to date remains the oscillation anomaly with the largest statistical significance, was later reinforced at lower significance by MiniBooNE [2]. Other experiments, in particular KARMEN [94], NOMAD [95], E776 [96], ICARUS [41, 97], and OPERA [40], have not been able to confirm the findings by LSND and MiniBooNE, albeit not ruling them out either. We summarize the data sets included in our analysis of $\nu_{e}$ and $\bar{\nu}_{e}$ appearance data in table 3 .

Compared to our previous publication, ref. [14], in which more technical details on our fits are given, we have added the following data sets:

1. New results from the ICARUS [41, 97] and OPERA [40] experiments in the high energy $(\sim 20 \mathrm{GeV})$ CNGS beam. Both experiments have searched for anomalous $\nu_{\mu} \rightarrow \nu_{e}$ appearance, but have not found any evidence. They are thus able to impose constraints over a wide range of $\Delta m_{41}^{2}$ values. 


\begin{tabular}{|lclr|}
\hline Experiment & References & Comments & Data points \\
\hline LSND & {$[1]$} & $\bar{\nu}_{\mu}$ from stopped pion source $(\mathrm{DaR})$ & 11 \\
LSND & {$[1]$} & combined DaR and DiF data $\left(\stackrel{(-)}{\nu}_{\mu} \rightarrow \stackrel{(-)}{\nu}_{e}\right)$ & N/A \\
MiniBooNE & {$[2,98]$} & $\nu_{\mu}$ and $\bar{\nu}_{\mu}$ from high-energy Fermilab beam & 22 \\
KARMEN & {$[94]$} & $\bar{\nu}_{\mu}$ from stopped pion source & 9 \\
NOMAD & {$[95]$} & $\nu_{\mu}$ from high-energy CERN beam & 1 \\
E776 & {$[96]$} & $\nu_{\mu}$ from high-energy Brookhaven beam & 24 \\
ICARUS & {$[41,97]$} & $\nu_{\mu}$ from high-energy CERN beam & 1 \\
OPERA & {$[40]$} & $\nu_{\mu}$ from high-energy CERN beam & 1 \\
\hline
\end{tabular}

Table 3. Experimental data sets included in our $\stackrel{(-)}{\nu}_{\mu} \rightarrow \stackrel{(-)}{\nu}_{e}$ analysis. For LSND, we have carried out analyses using only decay-at-rest $(\mathrm{DaR})$ data, or the combination with decay-in-flight $(\mathrm{DiF})$ data. In the latter case we use a $\chi^{2}$ table provided by the collaboration, which cannot be associated with a number of data points. The total number of data points in the appearance channel (when using LSND DaR data only) is 69.

2. Decay-in-flight data from LSND. The neutrino oscillation analysis of LSND is based on a search for anomalous $\bar{\nu}_{e}$ appearance in the neutrino flux from a stopped pion source. Since the LSND detector was placed downstream from the pion production target, it received not only $\nu_{\mu}, \bar{\nu}_{\mu}$, and $\nu_{e}$ from $\pi^{+}$decays at rest (DaR), but also neutrinos and anti-neutrinos from pions decaying in flight (DiF). A discussion of the impact of DiF data in the context of the global sterile neutrino fit can be found in ref. [99]. The LSND collaboration has kindly provided tabulated $\chi^{2}$ values from their combined $\mathrm{DaR}+\mathrm{DiF}$ fit. The LSND fit is based on the two-flavour approximation, so to include the tabulated $\chi^{2}$ values in our 4 -flavour analysis, we compute at each parameter point the effective two-flavour mixing angle

$$
\sin ^{2} 2 \theta_{\mu e} \equiv 4\left|U_{e 4}\right|^{2}\left|U_{\mu 4}\right|^{2}
$$

from the full four-flavour mixing matrix $U$. In the following, we will show results using both our previous fitting code that includes only DaR data as well as results based on the tabulated two-flavour $\chi^{2}$ values from LSND for DaR+DiF data.

Our results are plotted in figure 4, which shows the favoured parameter regions projected onto the $\sin ^{2} 2 \theta_{\mu e}-\Delta m_{41}^{2}$ plane. We see that all $\stackrel{(-)}{\nu}_{\mu} \rightarrow \stackrel{(-)}{\nu}_{e}$ data sets are consistent among each other: a large chunk of the parameter region favoured by LSND and MiniBooNE is not probed by any of the other searches. The strongest constraints come from OPERA at $\Delta m_{41}^{2} \lesssim 0.5 \mathrm{eV}^{2}$, and from KARMEN at larger $\Delta m_{41}^{2}$. Note that data from E776 is combined with solar neutrino data because a fit to E776 data alone would not be meaningful as it would leave possible oscillations of the $\nu_{e}$ and $\bar{\nu}_{e}$ backgrounds into sterile states unconstrained. Fitting E776 data jointly with solar neutrino data provides a reasonable constraint on $\left|U_{e 4}\right|$, cf. figure 3 .

The conclusions drawn from figure 4 agree qualitatively with the ones from our earlier paper ref. [14]. Some constraints, in particular those from OPERA and ICARUS, have 

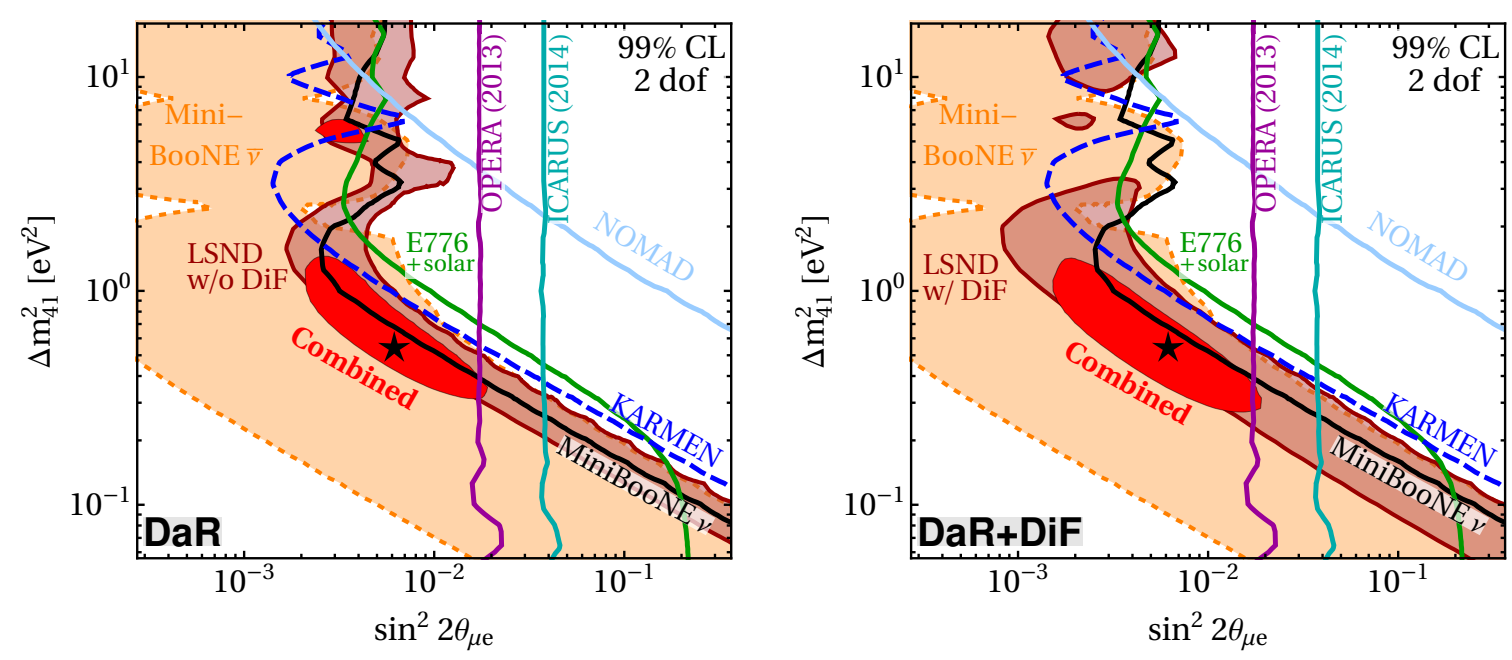

Figure 4. Constraints on short-baseline $\nu_{\mu} \rightarrow \nu_{e}$ and $\bar{\nu}_{\mu} \rightarrow \bar{\nu}_{e}$ oscillations in the presence of sterile neutrinos in $3+1$ scenarios. We show the allowed parameter regions, projected onto the plane spanned by the effective mixing angle $\sin ^{2} 2 \theta_{\mu e} \equiv 4\left|U_{e 4}\right|^{2}\left|U_{\mu 4}\right|^{2}$ and the mass squared difference $\Delta m_{41}^{2}$. In the left panel only decay-at-rest (DaR) data from LSND is included, while in the right panel also decay-in-flight data $(\mathrm{DiF})$ is used.

become significantly stronger and now disfavour values of $\sin ^{2} 2 \theta_{\mu e} \gtrsim 0.02$ that were still allowed previously. Note that our OPERA and ICARUS limits deviate slightly from those published by the respective collaborations [40, 41, 97] because we include oscillations of the backgrounds. Moreover, for consistency with the other exclusion curves in figure 4, we interpret the $\chi^{2}$ values from our OPERA and ICARUS fits assuming two degrees of freedom. We have checked that our code reproduces the official limits from refs. [40, 41, 97] very well when the same assumptions as in the official publications are used.

Let us mention that the global $\stackrel{(-)}{\nu}_{\mu} \rightarrow \stackrel{(-)}{\nu}_{e}$ analysis has a relatively poor goodness of fit. For the combined best fit point using the LSND DaR analysis we find $\chi_{\min }^{2} / \mathrm{dof}=$ $89.9 /(69-2)$, which corresponds to a $p$-value of $3.3 \%$. This is mostly driven by the MiniBooNE low-energy excess, which cannot be fitted well in the $3+1$ scenario, and by the contribution from E776 whose spectrum gives a relatively poor fit. This feature has been present also in our previous analysis [14], where a more detailed discussion can be found.

In all cases LSND dominates the appearance fit. LSND alone disfavours the nooscillation hypothesis with $\Delta \chi^{2}=44$ (29) when using DaR (DaR+DiF) data. For the combined appearance analysis these numbers increase slightly, due to the hint for appearance in MiniBooNE data. We find that the no-oscillation hypothesis for all appearance data is disfavoured compared to the best fit by $\Delta \chi^{2}=46$ (35) when using LSND DaR $(\mathrm{DaR}+\mathrm{DiF})$ data.

Comparing the allowed regions with and without the inclusion of decay-in-flight data in LSND, we see that the impact on the global fit is relatively minor. This is because although the LSND region with DiF data extends to slightly smaller values of $\sin ^{2} 2 \theta_{\mu e}$, MiniBooNE appearance data prefers smaller $\Delta m_{41}^{2}$ and mixing angles (especially for the neutrino mode data), somewhat limiting the impact of LSND DiF data when LSND and 
MiniBooNE data are combined. We observe only a slight broadening of the parameter regions preferred by LSND and by the combination of all $\nu_{\mu} \rightarrow \nu_{e}$ and $\bar{\nu}_{\mu} \rightarrow \bar{\nu}_{e}$ appearance data. We will see in section 7 that this slightly reduces the tension between appearance and disappearance data, but does not remove it.

\section{$5 \stackrel{(-)}{\nu}$, disappearance data}

Searches for muon neutrino disappearance due to oscillations involving a fourth neutrino mass state have recently received a significant boost thanks to novel results on sterile neutrinos from atmospheric neutrino data (both in the TeV energy window from IceCube [52] and at lower energy from DeepCore [49]) as well as from a combined analysis of MINOS and MINOS+ charged current (CC) and neutral current (NC) data [43]. Also $\mathrm{NO} \nu \mathrm{A}$ has presented a first search for sterile neutrinos based on NC data [44]. Searches for a deficit of NC events are of particular interest because they are sensitive to mixing of sterile neutrinos with any active neutrino flavor. As such, any deficit found would be a unique signature of sterile neutrinos. The new analyses by IceCube, DeepCore, MINOS/MINOS+, and $\mathrm{NO} \nu \mathrm{A}$ complement, and significantly extend, the exclusion regions from the short-baseline experiments CDHS [100] and MiniBooNE [101, 102], from Super-Kamiokande data on atmospheric neutrinos [48, 103], and from MINOS [104].

The high-energy IceCube analysis from ref. [52] exploits the fact that active-to-sterile neutrino oscillations in matter are resonantly enhanced by the MSW effect $[55,56]$ at an energy of

$$
E_{\mathrm{res}}=5.3 \mathrm{TeV} \times\left(\frac{5 \mathrm{~g} / \mathrm{cm}^{3}}{\rho_{\oplus}}\right)\left(\frac{\Delta m_{41}^{2}}{1 \mathrm{eV}^{2}}\right)
$$

Here $\rho_{\oplus}$ is the mass density of the material through which neutrinos are propagating. It is on average $\sim 3 \mathrm{~g} / \mathrm{cm}^{3}$ in the Earth's crust and outer mantle, $\sim 5 \mathrm{~g} / \mathrm{cm}^{3}$ in the inner mantle, and between 10 and $13 \mathrm{~g} / \mathrm{cm}^{3}$ in the core [105]. Equation (5.1) implies that, for sterile neutrinos at the $\mathrm{eV}$-scale, neutrino telescopes like IceCube can in principle observe maximal oscillations at $\mathrm{TeV}$ energies - a sweet spot well above the detection threshold, but still low enough for the atmospheric neutrino flux to be appreciable [57, 58]. For larger or smaller $\Delta m_{41}^{2}$, the sensitivity is expected to dwindle as the resonance moves to energies with a lower neutrino flux, or moves below the energy threshold of the detector. A limiting factor to this analysis is the fact that, for $\Delta m_{41}^{2}>0$ as considered here, the resonance is in the anti-neutrino sector. Since neutrino telescopes cannot distinguish neutrinos from anti-neutrinos on an event-by-event basis, and since anti-neutrino cross-sections are smaller by about a factor of three than neutrino cross-sections, the magnitude of the observable effect is reduced. ${ }^{3}$ Moreover, for small mixing angles, the resonance width,

$$
\Delta E_{\mathrm{res}} \sim \frac{\Delta m_{41}^{2} \sin ^{2} 2 \theta_{24}}{2 V_{\mathrm{MSW}}},
$$

\footnotetext{
${ }^{3}$ For $\Delta m_{41}^{2}<0$ the resonance would occur for neutrinos and the signal would therefore be stronger. However, such scenarios are in strong tension with cosmology.
} 
is small, so that only a very small fraction of the energy spectrum is affected. The narrow width, combined with the limited experimental energy resolution, further reduces the sensitivity of IceCube. In eq. (5.2), $V_{\mathrm{MSW}} \simeq 1.9 \times 10^{-14} \mathrm{eV} \times\left[\rho_{\oplus} /\left(\mathrm{g} / \mathrm{cm}^{3}\right)\right]$ is the neutral current-induced MSW potential for muon and tau neutrinos. Finally, systematic uncertainties play a crucial role in the analysis from ref. [52]. Technical details on our implementation of the IceCube analysis are given in appendix B.

In addition to the $\mathrm{TeV}$ neutrino events discussed above, the IceCube collaboration has also observed atmospheric neutrinos in the tens-of-GeV range through its sub-detector DeepCore. The information on sterile neutrinos which can be extracted from this lowenergy sample is very similar to that provided by Super-Kamiokande atmospheric data, which has been discussed in detail in refs. $[14,93]$. As explained there, low-energy atmospheric neutrino data can put a strong bound on $\left|U_{\mu 4}\right|^{2}$ through the suppression of the $P_{\mu \mu}$ oscillation probability which a mixing of $\nu_{\mu}$ with a heavy state would imply. Moreover, such data also constrains $\left|U_{\tau 4}\right|^{2}$ because the zenith-angle dependence of $P_{\mu \mu}$ is modified if oscillations driven by $\Delta m_{31}^{2}$ deviate from vacuum-like $\nu_{\mu} \rightarrow \nu_{\tau}$ oscillations. The formalism for neutrino oscillations discussed in appendix D of ref. [14] for Super-Kamiokande phase 1-3 data is also applied here to phase 4 results as well as to DeepCore data.

The MINOS detector is particularly interesting for sterile neutrino searches as it has observed neutrino oscillations over a fairly wide range of energies: during the original MINOS run, the NuMI beam was tuned to a peak energy of $\sim 2 \mathrm{GeV}$, while in the MINOS+ phase, the peak energy was at about $6 \mathrm{GeV}$, with the spectrum extending to tens of GeV. Moreover, the MINOS collaboration has analysed not only $\mathrm{CC} \nu_{\mu}$ disappearance sensitive mainly to $U_{\mu 4}$, but has also searched for disappearance in NC events. Since MINOS/MINOS+ has near and far detectors, the experiment is sensitive over a wide range of $\Delta m_{41}^{2}$ values. For $\Delta m_{41}^{2} \sim 10^{-3}-10^{-1} \mathrm{eV}^{2}$, an oscillation pattern can be observed in the far detector, while no oscillations are expected in the near detector. At larger mass squared difference, oscillations in the far detector enter the averaging regime. At $\Delta m_{41}^{2} \sim 1-100 \mathrm{eV}^{2}$, oscillation patterns begin to emerge in the near detector. In our analysis of MINOS/MINOS+ data, we follow very closely the recommendations accompanying the MINOS/MINOS+ data release [43]. ${ }^{4}$

We have also implemented the $\mathrm{NO} \nu \mathrm{A}$ neutral current analysis from ref. [44]. Due to the low number of events and the difficult reconstruction of the neutrino energy in $\mathrm{NC}$ events, only total rates are used in the analysis. The dominant background in this analysis are misidentified charged current events. Following ref. [44], we implement a $12.2 \%(15.3 \%)$ systematic uncertainty on the signal (background) rates. Compared to the MINOS/MINOS+ NC search, the narrow-band beam employed in $\mathrm{NO} \nu \mathrm{A}$ means that

\footnotetext{
${ }^{4}$ The validity of the MINOS/MINOS+ analysis from ref. [43] has been called into question in ref. [106] based on the fact that the limit on $U_{\mu 4}$ is surprisingly strong at large $\Delta m_{41}^{2}$, while on the other hand a per cent level deviation from unity in the far/near ratio of $\mathrm{NC}$ events indicates non-negligible systematic bias. We have checked that a more conservative MINOS/MINOS+ fit with a completely free flux normalization would indeed significantly deteriorate the limit at large $\Delta m_{41}^{2}$. However, we have also checked that the impact of a free normalization would be very small at $\Delta m_{41}^{2}$ in the region relevant to the global fit. We will also show in section 7 that even removing MINOS/MINOS+ completely from our fit would not change our conclusions. Therefore, all results presented below will be based on the official MINOS/MINOS+ fit from the data release accompanying ref. [43].
} 


\begin{tabular}{|lclr|}
\hline Experiment & References & Comments & Data points \\
\hline IceCube (IC) & {$[52-54]$} & MSW resonance in high- $E$ atmospheric $\bar{\nu}_{\mu}$ & 189 \\
CDHS & {$[100]$} & accelerator $\nu_{\mu}$ & 15 \\
MiniBooNE & {$[101,102,107]$} & accelerator $\nu_{\mu}$ and $\bar{\nu}_{\mu}$ & $15+42$ \\
Super-Kamiokande (SK) & {$[48,103]$} & low- $E$ atmospheric neutrinos & 70 \\
DeepCore (DC) & {$[49,50]$} & low- $E$ atmospheric neutrinos & 64 \\
NO $\nu$ A & {$[44]$} & NC data & 1 \\
MINOS/MINOS + & {$[43]$} & accelerator $\nu_{\mu}$, CC \& NC event spectra & 108 \\
\hline
\end{tabular}

Table 4. Experimental data sets included in our $\stackrel{(-)}{\nu}_{\mu} \rightarrow \stackrel{(-)}{\nu}_{\mu}$ disappearance analysis. The total number of data points in this channel is 504 .

the experiment is sensitive to a much smaller range of $\Delta m_{41}^{2}$ values, namely between $0.05 \mathrm{eV}^{2}$ and $0.5 \mathrm{eV}^{2}$. Even in this mass range, the $\mathrm{NO} \nu \mathrm{A}$ search for sterile neutrinos is not competitive with other searches yet as it is suffers from large systematic uncertainties related to detector modelling and energy reconstruction, but it is expected to improve considerably in the future.

We summarize the $\nu_{\mu} / \bar{\nu}_{\mu}$ disappearance data sets included in our analysis in table 4 . Details on the CDHS and MiniBooNE analyses are given in ref. [14] and in the references therein. Our results are shown in figure 5 as a function of the mixing matrix element $\left|U_{\mu 4}\right|^{2}$ and the mass squared difference $\Delta m_{41}^{2}$. The plot reveals strong limits of order $\left|U_{\mu 4}\right|^{2} \lesssim 10^{-2}$ across a wide range of $\Delta m_{41}^{2}$ values from $\sim 2 \times 10^{-1} \mathrm{eV}^{2}$ to $\sim 10 \mathrm{eV}^{2}$. MINOS/MINOS+ gives an important contribution in most of the parameter space. The strong constraint from atmospheric neutrino data at $\Delta m_{41}^{2} \lesssim 1 \mathrm{eV}^{2}$ is dominated by IceCube. At large masses, MiniBooNE and to some extent CDHS are competitive with the MINOS/MINOS+ bound. Comparing to the parameter region preferred by appearance and $\nu_{e} / \bar{\nu}_{e}$ disappearance data (which includes the oscillation anomalies), we see dramatic tension. Given the constraints on $U_{e 4}$ from reactor experiments, the values of $\sin ^{2} 2 \theta_{\mu e} \equiv 4\left|U_{e 4}\right|^{2}\left|U_{\mu 4}\right|^{2}$ required by LSND and MiniBooNE can only be reached if $\left|U_{\mu 4}\right|$ is large. This, however, is clearly disfavoured by multiple $\nu_{\mu} / \bar{\nu}_{\mu}$ disappearance null results. This is the origin of the severe tension in the global fit we are going to report below. As we are going to discuss, this tension has become very robust and does not rely on any single $\stackrel{(-)}{\nu}_{\mu}$ disappearance data set.

\section{Constraints on $\left|U_{\tau 4}\right|$}

Mixing between tau neutrinos and possible sterile states is particularly difficult to constrain since no $\nu_{\tau}$ sources are available. Nevertheless, constraints can be obtained in the following two ways: (i) studying matter effects. All active neutrino flavors experience an MSW potential caused by coherent forward scattering through $Z$ boson exchange, while sterile neutrinos do not. This influences $\nu_{e}$ disappearance observed in solar neutrino experiments, as well as $\nu_{\mu}$ disappearance observed in beam experiments and in atmospheric neutrinos. The latter yield particularly strong limits as they possess the longest baselines in matter. (ii) exploiting neutral current events, which are sensitive to any disappearance of active 


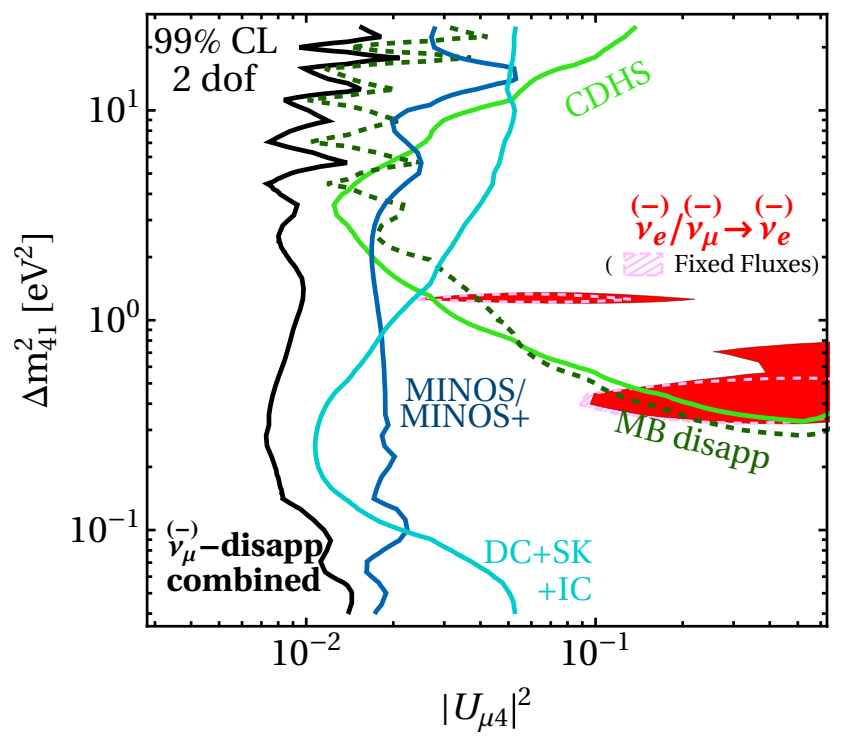

Figure 5. Constraints on the $3+1$ scenario from $\nu_{\mu} / \bar{\nu}_{\mu}$ disappearance. We show the allowed parameter regions, projected onto the plane spanned by the mixing matrix element $\left|U_{\mu 4}\right|^{2}$ and the mass squared difference $\Delta m_{41}^{2}$. Note that the exclusion limit from NO $\nu \mathrm{A}$ is still too weak to appear in the plot. It is, however, included in the curve labelled "combined", which includes all data listed in table 4. The curve labelled $\mathrm{DC}+\mathrm{SK}+\mathrm{IC}$ combines all our atmospheric neutrino data; for this bound we have fixed the parameters $\theta_{12}, \theta_{13}, \theta_{14}$ but minimize with respect to all other mixing parameters, including complex phases. For comparison, we also show the parameter region favoured by $\nu_{e}$ disappearance and $\nu_{\mu} \rightarrow \nu_{e}$ appearance data (using LSND DaR+DiF), projected onto the $\left|U_{\mu 4}\right|^{2}-\Delta m_{41}^{2}$ plane; we show the allowed regions for the analyses with fixed and free reactor neutrino fluxes.

neutrinos. This approach allows us to derive constraints from the sterile neutrino searches in MINOS/MINOS+ [43] and NO $\nu \mathrm{A}$ [44], and from SNO solar neutrino data [79-81]. The corresponding analysis codes used in our fit are the same as discussed in sections 3 and 5. Compared to ref. [14], we have in particular added IceCube, DeepCore, MINOS/MINOS+, and $\mathrm{NO} \nu \mathrm{A}$ data to the fit.

Our results are shown in the four panels of figure 6. Each panel corresponds to a different fixed value of $\Delta m_{41}^{2}$, and the corresponding contours have been drawn based on the $\chi^{2}$ differences relative to the best fit point for this fixed $\Delta m_{41}^{2}$. The difference in $\chi^{2}$ between the individual best fit points and the global one are, however, very small, as indicated in each panel. The reason is that in all cases the best fit point is very close to zero mixing, and therefore has very similar $\chi^{2}$ values. In defining the exclusion contours we have assumed a $\chi^{2}$ distribution with two degrees of freedom. We see that depending on $\Delta m_{41}^{2}$, the limit on $\left|U_{\mu 4}\right|$ is driven by MINOS/MINOS+, IceCube, or the short-baseline experiments MiniBooNE and CDHS, in agreement with figure 5. The strongest constraints on $\left|U_{\tau 4}\right|$ typically come from atmospheric neutrinos. We find that the combined bound is independent of $\Delta m_{41}^{2}$ and is given by

$$
\left|U_{\tau 4}\right|^{2}<0.13(0.17) \quad \text { at } \quad 90 \%(99 \%) \mathrm{CL} .
$$



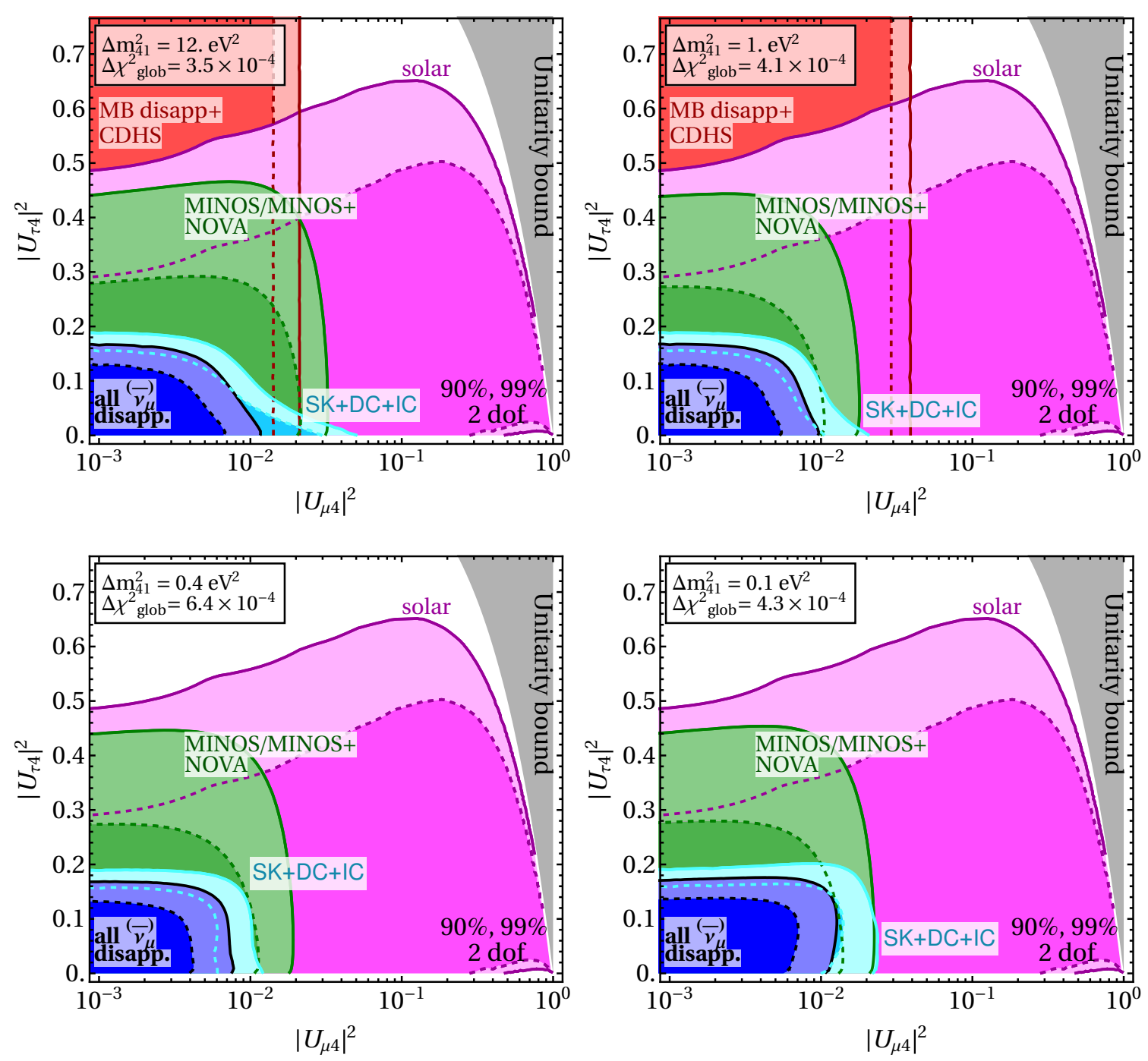

Figure 6. Constraints on the mixing of sterile neutrinos with muon and tau neutrinos, parameterized by the corresponding elements $\left|U_{\mu 4}\right|$ and $\left|U_{\tau 4}\right|$ of the leptonic mixing matrix. In each panel, $\Delta m_{41}^{2}$ has been fixed to a different value, while $\Delta m_{31}^{2}, \theta_{23}, \theta_{12}$ and $\theta_{14}$, as well as complex phases have been profiled out in those experiments where they have a significant impact. Exclusion contours are drawn relative to the minimum $\chi^{2}$ in each panel; the difference to the global minimum $\chi^{2}$ is indicated in each plot. Grayed out areas show the parameter region incompatible with the unitarity of the leptonic mixing matrix.

Let us mention that recently ref. [108] has found a $2 \sigma$ hint from Ice Cube data in favour of sterile neutrinos with non-zero $\nu_{4}-\nu_{\tau}$ mixing in the high-mass region, with $\Delta m_{41}^{2} \simeq$ $100 \mathrm{eV}^{2}$. With our code we cannot reproduce their results and we do not find any hint for sterile neutrino mixing in that mass range. The origin of these different results is currently under investigation. 


\begin{tabular}{|llrl|}
\hline Data set & Reference & Data points & Relevant parameters \\
\hline$\stackrel{(-)}{\nu}_{e}$ disappearance & Table 1 & 594 & $\Delta m_{31}^{2}, \Delta m_{41}^{2}, \theta_{12}, \theta_{14}, \theta_{24}, \theta_{34}$ \\
$\left(\stackrel{-}{\nu}_{\mu}\right.$ disappearance & Table 4 & 504 & $\Delta m_{31}^{2}, \Delta m_{41}^{2}, \theta_{23}, \theta_{14}, \theta_{24}, \theta_{34}$ \\
$\stackrel{(-)}{\nu}_{\mu} \rightarrow \stackrel{(-)}{\nu}_{e}$ appearance (w/o LSND DiF) & Table 3 & 69 & $\Delta m_{41}^{2},\left|U_{e 4} U_{\mu 4}\right|$ \\
\hline Total number of data points: & \multicolumn{3}{l}{} \\
\hline
\end{tabular}

Table 5. Number of degrees of freedom and parameters relevant to the counting of degrees of freedom for each data set. More details on the individual experiments are given in the corresponding tables. The number of degrees of freedom for the LSND decay-in-flight analysis is not available. Thus, in the sum of degrees of freedoms for appearance and all data sets, we used the LSND decay-at-rest number. See text for details and comments on additional nuisance parameters.

\section{The disappearance-appearance tension}

As discussed above, results on the $\nu_{e} \rightarrow \nu_{e}, \nu_{\mu} \rightarrow \nu_{e}$, and $\nu_{\mu} \rightarrow \nu_{\mu}$ oscillation channels (and the corresponding anti-neutrino modes) over-constrain $\mathrm{eV}$-scale sterile neutrino models. The reason can be easily understood by going to the short-baseline limit in which baselines are so short that oscillations induced by $\Delta m_{31}^{2}$ and $\Delta m_{21}^{2}$ did not yet develop. In this limit, eqs. (2.3) and (2.4) show that the bounds on $\left|U_{e 4}\right|$ and $\left|U_{\mu 4}\right|$ from electron and muon disappearance data lead to a quadratic suppression of the effective amplitude $\sin ^{2} 2 \theta_{e \mu}$, eq. (4.1), relevant for $\nu_{\mu} \rightarrow \nu_{e}$ appearance [109-111]. Thus constraints from disappearance data challenge an explanation of the anomalies in the appearance channel in terms of sterile neutrino oscillations. While this tension has persisted for a very long time, see for instance ref. [99], it has become exceedingly severe with recent data, rendering the sterile neutrino hypothesis as an explanation for the appearance anomalies very unlikely, see below.

The results of the combined fit are summarized in table 6 , which shows the results for $\stackrel{(-)}{\nu}_{e}$ disappearance, $\stackrel{(-)}{\nu}_{\mu}$ disappearance, and $\stackrel{(-)}{\nu}_{e}$ appearance data separately as well as combined. The total numbers of data points in these analyses are summarized in table 5 . The last column of that table also indicates which parameters need to be considered when counting degrees of freedom. For the $\stackrel{(-)}{\nu}_{\mu}$ disappearance data we do take into account complex phases in the fit [14], but since numerically their effect is very small we do not count them as full dof. We do, however, treat the normalization of the atmospheric neutrino flux as a free parameter in the IceCube analysis. Concerning the appearance sample, for most of the data summarized in table 3 the short-baseline approximation holds, motivating the use of only the effective mixing angle quoted in table 5. Exceptions are the long-baseline experiments ICARUS and OPERA, which depend on more parameters, but play a role neither for the appearance best fit point nor for the global best fit point. Therefore, we consider only two effective parameters for the appearance sample. For the global analysis we count seven parameters plus the IceCube global normalization. The reactor analysis with free fluxes has two additional free parameters.

We would now like to quantify the tension between different subsets of the global data that is evident from figure 5 . We first note that combining all data sets we find a goodnessof-fit for the global best fit point around $65 \%$, see table 6 . This good $p$-value does not 


\begin{tabular}{|c|c|c|c|c|c|c|c|}
\hline Analysis & $\Delta m_{41}^{2}\left[\mathrm{eV}^{2}\right]$ & $\left|U_{e 4}\right|$ & $\left|U_{\mu 4}\right|$ & $\chi_{\min }^{2} /$ dof & GOF & $\chi_{\mathrm{PG}}^{2}$ & PG \\
\hline appearance (DaR) & 0.573 & $4\left|U_{e 4}\right|$ & $=6.97 \times 10^{-3}$ & $89.8 / 67$ & $3.3 \%$ & & \\
\hline appearance (DiF) & 0.559 & $4\left|U_{e 4}\right|$ & $=6.31 \times 10^{-3}$ & $79.1 /-$ & & & \\
\hline$\stackrel{(-)}{\nu}_{\mu}$ disapp & $2 \times 10^{-3}$ & 0.12 & 0.039 & $468.9 / 497$ & $81 \%$ & & \\
\hline \multicolumn{8}{|c|}{ Reactor fluxes fixed at predicted value \pm quoted uncertainties } \\
\hline$\stackrel{(-)}{\nu}$ disapp & 1.3 & 0.1 & - & $552.8 / 588$ & $85 \%$ & & \\
\hline Global (DiF) & 6.03 & 0.2 & 0.1 & $1127 /-$ & & 25.7 & $2.6 \times 10^{-6}$ \\
\hline Global (DaR) & 5.99 & 0.21 & 0.12 & $1141 / 1159$ & $64 \%$ & 28.9 & $5.3 \times 10^{-7}$ \\
\hline \multicolumn{8}{|c|}{ Reactor fluxes floating freely } \\
\hline$\stackrel{(-)}{\nu}_{e}$ disapp & 1.3 & 0.095 & - & $542.9 / 586$ & $90 \%$ & & \\
\hline Global (DiF) & 6.1 & 0.20 & 0.10 & $1121 /-$ & & 29.6 & $3.7 \times 10^{-7}$ \\
\hline Global (DaR) & 6.0 & 0.22 & 0.11 & $1134 / 1157$ & $68 \%$ & 32.1 & $1.1 \times 10^{-7}$ \\
\hline
\end{tabular}

Table 6. Parameter values at the global best fit point and at the best fit points obtained for subsets of the data. We also indicate the $\chi^{2}$ per degree of freedom at the best fit points, as well as the corresponding goodness-of-fit values. The numbers of data points, and the parameters relevant to the counting of degrees of freedom are summarized in table 5. For the global fit, we also indicate the results of the parameter goodness-of-fit test [92] comparing appearance to disappearance data. The labels "DaR" and "DiF" refer to the LSND analysis employed, where "DiF" implies the joint use of DaR+DiF data, see section 4. Note that, as the number of degrees of freedom for the LSND DiF data is not available, we do not list the corresponding goodness of fit values.

reflect the tension we found because many data points entering the global fit have only little sensitivity to sterile neutrino oscillations, thus diluting the power of a goodness-of-fit test based on $\chi^{2} /$ dof.

A more reliable method for quantifying the compatibility of different data sets is the parameter goodness-of-fit (PG) test [92], which measures the penalty in $\chi^{2}$ that one has to pay for combining data sets, see appendix A for a brief review of this test. If the global neutrino oscillation data were consistent when interpreted in the framework of a $3+1$ model, any slicing into two statistically independent data sets $A$ and $B$ should result in an acceptable $p$-value from the PG test. To illustrate an inconsistency in the data, it is however sufficient to demonstrate that at least one way of dividing it leads to a poor value. Here, we choose to split the data into disappearance data encompassing the oscillation channels $\stackrel{(-)}{\nu}_{e} \rightarrow \stackrel{(-)}{\nu}_{e}$ and $\stackrel{(-)}{\nu}_{\mu} \rightarrow \stackrel{(-)}{\nu}_{\mu}$, and appearance data covering the $\stackrel{(-)}{\nu}_{\mu} \rightarrow \stackrel{(-)}{\nu}_{e}$ channel. Note that it is important to chose data sets independent of their "result". For instance, dividing data into "evidence" and "no-evidence" samples would bias the PG test.

The tension between appearance and disappearance data is shown graphically in figure 7. The figure illustrates the lack of overlap between the parameter region favoured by appearance data (driven by LSND and MiniBooNE) and the strong exclusion limits from disappearance data. The tension persists independently of whether reactor fluxes are fixed or kept free, and whether the LSND DaR or DaR+DiF samples are used. The corresponding results from the PG test are shown in the last two columns of table 6 . To evaluate the $p$-value of the PG test statistic we use two degrees of freedom, corresponding to the two parameters in common to appearance and disappearance data, see table 5 and the related 


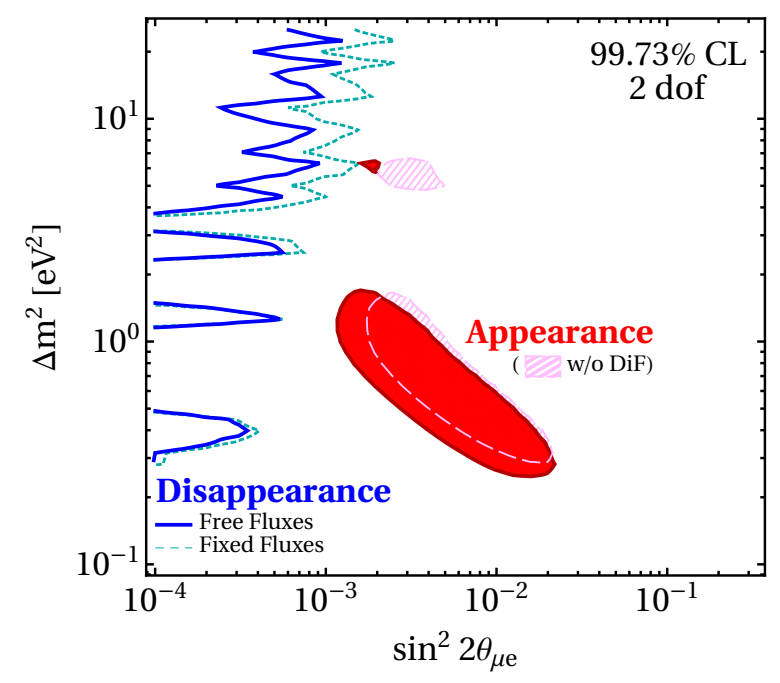

Figure 7. Appearance versus disappearance data in the plane spanned by the effective mixing angle $\sin ^{2} 2 \theta_{\mu e} \equiv 4\left|U_{e 4} U_{\mu 4}\right|^{2}$ and the mass squared difference $\Delta m_{41}^{2}$. The blue curves show limits from the disappearance data sets using free reactor fluxes (solid) or fixed reactor fluxes (dashed), while the shaded contours are based on the appearance data sets using LSND DaR+DiF (red) and LSND DaR (pink hatched). All contours are at 99.73\% CL for 2 dof.

discussion. We observe that for none of the analyses given in the table, the $p$-value for appearance and disappearance data being consistent exceeds $10^{-5}$, with the "best" compatibility of $p=2.6 \times 10^{-6}$ emerging for fixed reactor fluxes and using LSND DaR+DiF data. We conclude that the appearance/disappearance tension excludes a sterile neutrino oscillation explanation of the $\stackrel{(-)}{\nu}_{\mu} \rightarrow \stackrel{(-)}{\nu}_{e}$ anomalies at the $4.7 \sigma$ level.

Note that the parameter goodness-of-fit for the analysis using free reactor fluxes is worse than the one for fixed reactor fluxes. The reason can be understood from the $\chi^{2}$ numbers given in table 6 . We see that the $\chi_{\text {min }}^{2}$ of $\stackrel{(-)}{\nu}_{e}$ disappearance decreases by more (9.9 units) than the global best fit point (7 or 6 units for DaR or DaR+DiF, respectively), when leaving reactor fluxes free. Therefore, reactor data alone benefits more from free fluxes than the appearance/disappearance tension, which increases the $\chi^{2}$ penalty to pay for the combination in the case of free fluxes.

In table 7 we investigate the robustness of the appearance/disappearance tension. We show how the PG would improve if individual experiments or classes of experiments were removed from the fit. We stress that we are not aware of any strong reason to discard data from particular experiments. The sole purpose of this exercise is to demonstrate the impact of individual data sets and establish the robustness of our conclusion.

The first row in table 7 corresponds to the global analysis using free reactor fluxes and LSND DaR+DiF data, which is the combination of data we use throughout this table. The remaining part of the table shows that very strong tension remains even after removing any individual experiment. In particular, the PG remains below $\approx 5 \times 10^{-6}$ when any of the $\stackrel{(-)}{\nu}_{\mu}$ disappearance data sets are removed, so it does not rely on the particular treatment of any of those experiments. Even when all reactor data are removed, the PG remains very small $\left(3.8 \times 10^{-5}\right)$. 


\begin{tabular}{|c|c|c|c|c|c|c|c|}
\hline Analysis & $\chi_{\text {min,global }}^{2}$ & $\chi_{\text {min,app }}^{2}$ & $\Delta \chi_{\text {app }}^{2}$ & $\chi_{\min , \text { disapp }}^{2}$ & $\Delta \chi_{\text {disapp }}^{2}$ & $\chi_{\mathrm{PG}}^{2} / \mathrm{dof}$ & $\mathrm{PG}$ \\
\hline Global & 1120.9 & 79.1 & 11.9 & 1012.2 & 17.7 & $29.6 / 2$ & $3.71 \times 10^{-7}$ \\
\hline \multicolumn{8}{|c|}{ Removing anomalous data sets } \\
\hline w/o LSND & 1099.2 & 86.8 & 12.8 & 1012.2 & 0.1 & $12.9 / 2$ & $1.6 \times 10^{-3}$ \\
\hline w/o MiniBooNE & 1012.2 & 40.7 & 8.3 & 947.2 & 16.1 & $24.4 / 2$ & $5.2 \times 10^{-6}$ \\
\hline $\mathrm{w} / \mathrm{o}$ reactors & 925.1 & 79.1 & 12.2 & 833.8 & 8.1 & $20.3 / 2$ & $3.8 \times 10^{-5}$ \\
\hline $\mathrm{w} / \mathrm{o}$ gallium & 1116.0 & 79.1 & 13.8 & 1003.1 & 20.1 & $33.9 / 2$ & $4.4 \times 10^{-8}$ \\
\hline \multicolumn{8}{|l|}{ Removing constraints } \\
\hline w/o IceCube & 920.8 & 79.1 & 11.9 & 812.4 & 17.5 & $29.4 / 2$ & $4.2 \times 10^{-7}$ \\
\hline w/o $\operatorname{MINOS}(+)$ & 1052.1 & 79.1 & 15.6 & 948.6 & 8.94 & $24.5 / 2$ & $4.7 \times 10^{-6}$ \\
\hline w/o MB disapp & 1054.9 & 79.1 & 14.7 & 947.2 & 13.9 & $28.7 / 2$ & $6.0 \times 10^{-7}$ \\
\hline w/o CDHS & 1104.8 & 79.1 & 11.9 & 997.5 & 16.3 & $28.2 / 2$ & $7.5 \times 10^{-7}$ \\
\hline \multicolumn{8}{|c|}{ Removing classes of data } \\
\hline$\stackrel{(-)}{\nu}_{e}$ dis vs app & 628.6 & 79.1 & 0.8 & 542.9 & 5.8 & $6.6 / 2$ & $3.6 \times 10^{-2}$ \\
\hline$\stackrel{(-)}{\nu}_{\mu}$ dis vs app & 564.7 & 79.1 & 12.0 & 468.9 & 4.7 & $16.7 / 2$ & $2.3 \times 10^{-4}$ \\
\hline$\stackrel{(-)}{\nu}_{\mu}$ dis + solar vs app & 884.4 & 79.1 & 13.9 & 781.7 & 9.7 & $23.6 / 2$ & $7.4 \times 10^{-6}$ \\
\hline
\end{tabular}

Table 7. Results of the parameter goodness-of-fit (PG) test [92] comparing appearance to disappearance data. In this table we use the reactor flux-free analysis and LSND DaR+DiF data; therefore we do not quote dof for the $\chi^{2}$ values. The first row corresponds to the global fit, while the other row show the impact of removing individual experiments or sets of experiments from the fit. In columns 2-8, we list the $\chi^{2}$ at the global best fit point $\left(\chi_{\text {min,global }}^{2}\right)$, the $\chi^{2}$ at the appearance best fit $\left(\chi_{\min , a p p}^{2}\right)$, the difference in $\chi_{\text {app }}^{2}$ between the appearance best fit point and the global best fit point $\left(\Delta \chi_{\text {app }}^{2}\right)$, the $\chi^{2}$ at the disappearance best fit $\left(\chi_{\text {min,disapp }}^{2}\right)$, the difference in $\chi_{\text {disapp }}^{2}$ between the disappearance best fit point and the global best fit point $\left(\Delta \chi_{\text {disapp }}^{2}\right)$, the $\chi^{2}$ per dof for the PG test $\left(\chi_{\mathrm{PG}}^{2} /\right.$ dof, computed according to eq. (A.1)), and the resulting $p$-value given by eq. (A.3).

The only significant improvement is obtained when removing LSND. The still somewhat low PG of $0.16 \%$ is a manifestation of the tension between the MiniBooNE excess and the disappearance data. But it is clear that the very strong appearance/disappearance tension is driven by LSND. Note also that this remains true when MiniBooNE is removed, and therefore the result does not depend on the low-energy excess in MiniBooNE.

The only way to reconcile LSND would be to discard $\stackrel{(-)}{\nu}_{\mu}$ disappearance data altogether. Note that even if we remove all $\stackrel{(-)}{\nu})$ disappearance data, the PG remains low, at $2.4 \times$ $10^{-4}$. The reason is the non-trivial constraint on $\left|U_{e 4}\right|$ from the data sample we call $\stackrel{(-)}{\nu}_{\mu}$ disappearance (defined in table 4), see figure 3. Remarkably, just using $\stackrel{(-)}{\nu}_{\mu}$ disappearance plus solar neutrinos pushes the PG already to $7.4 \times 10^{-6}$. This demonstrates once again that our conclusion is independent of reactor neutrino data.

We observe from table 7 that the PG gets nearly an order of magnitude worse when removing the gallium data. The reason is the slight tension between gallium and reactor data discussed in section 3.2. If gallium is removed, the $\stackrel{(-)}{\nu}_{e}$ disappearance fit alone improves, and therefore the tension with appearance data increases.

Finally, we have also performed a slightly different PG test, by dividing the data into $\nu_{\mu}$ disappearance versus the combined $\nu_{e}$ appearance and $\nu_{e}$ disappearance data. This 
corresponds to the samples compared in figure 5. Using LSND DaR+DiF data and free reactor fluxes we obtain a $\chi_{\mathrm{PG}}^{2}=23.4$. According to table 5 , the common parameters in those two data sets are $\Delta m_{31}^{2}, \Delta m_{41}^{2}, \theta_{14}, \theta_{24}, \theta_{34}$. Therefore, $\chi_{\mathrm{PG}}^{2}$ has to be evaluated for 5 dof, leading to a $p$-value of $2.8 \times 10^{-4}$.

\section{Discussion and conclusions}

We have presented an updated global analysis of neutrino oscillation data within a $3+1$ sterile neutrino mass scheme. We have obtained two main results, which can be summarized as follows:

1. Reactor neutrino data show a $\gtrsim 3 \sigma$ preference for sterile neutrino oscillations with $\Delta m_{41}^{2} \approx 1.3 \mathrm{eV}^{2}$ and $\left|U_{e 4}\right| \approx 0.1$. This is driven by recent data from DANSS and NEOS and is based only on the relative comparisons of measured energy spectra and is therefore independent of predictions for the reactor neutrino fluxes and spectra. If flux predictions are taken into account, the preference for sterile neutrino oscillations in global $\stackrel{(-)}{\nu})$ disappearance data increases to $3.8 \sigma$.

2. Constraints on $\stackrel{(-)}{\nu}_{\mu}$ disappearance have become exceedingly strong, due to recent data from MINOS/MINOS+ and IceCube. This leads to very strong tension between the anomalies in the appearance sector (LSND and MiniBooNE) and disappearance data. We find that appearance and disappearance data are incompatible, with a parameter goodness-of-fit test yielding a $p$-value of less than $2.6 \times 10^{-6}$. This result does not rely on any single experiment in the $\stackrel{(-)}{\nu}_{\mu}$ sector and is robust with respect to theoretical predictions of reactor fluxes; the $p$-value remains at $3.8 \times 10^{-5}$ even if all reactor data are removed. The tension is dominated by LSND; the MiniBooNE anomaly plays a subleading role.

Our results rule out the sterile neutrino oscillation hypothesis as an explanation of the LSND and MiniBooNE anomalies, but it remains a viable option for the reactor and gallium anomalies.

Some comments are in order. Our conclusion in item 1 above is largely based on preliminary data from DANSS presented at conferences $[25,26]$. Our results are in agreement with another recent analysis done outside the DANSS collaboration [91]. However, those results will need to be supported by an official publication by the collaboration.

Throughout this work we have restricted ourselves to the $3+1$ scenario, adding just one mass state at the $\mathrm{eV}$ scale. However, we expect that the tension between appearance and disappearance data cannot be resolved by adding more sterile neutrinos. This has been quantitatively investigated previously, e.g. [14, 93]. There, it had been shown that adding more neutrinos does not relax the tension. The reason is that the quadratic suppression of the $\nu_{\mu} \rightarrow \nu_{e}$ oscillation amplitudes by constraints on the elements $\left|U_{e i}\right|$ and $\left|U_{\mu i}\right|(i \geq 4)$ from disappearance data remains equally true in scenarios with more than one $\mathrm{eV}$-scale mass states. Therefore we expect that our conclusion concerning the sterile neutrino explanation of appearance anomalies remains qualitatively true also for more sterile neutrinos. 
Finally, we remind the reader that a completely orthogonal set of constraints on $\mathrm{eV}$ scale sterile neutrinos comes from cosmology. The standard picture is that active neutrinos evolve into a superposition of active and sterile states at temperatures $\gtrsim \mathrm{MeV}$. Hard, flavour-sensitive collisions mediated by $W$ and $Z$ bosons collapse these superpositions into purely active or purely sterile states, with the relative probability given by the activesterile mixing angles. After a large number of collisions, active and sterile neutrinos come into thermal equilibrium. Because of this, the vanilla $3+1$ model appears to be strongly disfavoured by constraints on the number of relativistic species $N_{\text {eff }}$ at the time of Big Bang Nucleosynthesis (BBN) [112] and during the recombination epoch [113]. Moreover, constraints on the sum of neutrino masses, $\sum m_{\nu}$ from Cosmic Microwave Background and structure formation data disfavour extra neutrino species with masses $\gtrsim 0.3 \mathrm{eV}$ [113]. However, these constraints are model-dependent, and in non-minimal scenarios they can be weakened or absent. A full review of such scenarios is well beyond the scope of this work, therefore we only mention a few exemplary ones: in particular, mechanisms discussed in the literature include new interactions in the sterile sector [114-117], an extremely low reheating temperature [118], large neutrino-anti-neutrino asymmetries [119], late entropy production [120], and the presence of matter and antimatter domains during BBN [121]. It is also worth noting that the prevailing tension between local and cosmological determinations of the Hubble constant would be relaxed if $N_{\text {eff }}$ is somewhat larger than in the SM [122].

Note added. After the completion of this work new short-baseline data appeared. The STEREO and PROSPECT collaborations announced first results from their search for reactor neutrino disappearance [123, 124]. While no hint for oscillations has been found, their limits are still too weak to constrain our preferred regions shown in figures 2 and 3 and therefore we expect that the results of our global reactor analysis remain qualitatively unchanged.

MiniBooNE has confirmed their hint for $\nu_{\mu} \rightarrow \nu_{e}$ appearance with a significant of $4.5 \sigma$ [125]. This implies that the joint MiniBooNE/LSND significance for appearance becomes $6.1 \sigma$ [125]. We have performed a preliminary analysis of the new MiniBooNE data and found that (a) the joint allowed region for appearance data shown in figure 4 is hardly affected by the new data, and (b) the PG values for the consistency of appearance and disappearance data given in table 6 remain very similar. The reason is that while the MiniBooNE results increase the significance of oscillations, the $\Delta \chi^{2}$ from appearance data of the global best fit point remains similar to the previous data set.

\section{Acknowledgments}

We are grateful to Carlos Argüelles, Janet Conrad, and William Louis for useful discussions. We thank Gavin Davies for help in implementing the NO $\nu$ A results. The work of JK and MD has been supported by the German Research Foundation (DFG) under Grant Nos. KO 4820/1-1, FOR 2239, EXC-1098 (PRISMA) and by the European Research Council (ERC) under the European Union's Horizon 2020 research and innovation programme 
(grant agreement No. 637506, " $\nu$ Directions"). The work of MM and IMS has been supported by the Spanish government through MINECO/FEDER-UE grants FPA2015-65929$\mathrm{P}$ and FPA2016-78645-P, as well as the "Severo Ochoa" program grant SEV-2016-0597 of IFT. This project has received support from the European Union's Horizon 2020 research and innovation programme under the Marie Skłodowska-Curie grant agreements No. 690575 (InvisiblesPlus) and No. 674896 (Elusives). This manuscript has been authored by Fermi Research Alliance, LLC under Contract No. DE-AC02-07CH11359 with the U.S. Department of Energy, Office of Science, Office of High Energy Physics.

\section{A The parameter goodness-of-fit test}

In this appendix we briefly review the parameter goodness-of-fit (PG) test [92], which measures the compatibility of sub-sets of a data set. Let us subdivide the global data into two statistically independent sets $A$ and $B$. Let $\chi_{\min , A}^{2}$ and $\chi_{\min , B}^{2}$ be the minimum $\chi^{2}$ values obtained from individual fits to the two data sets, and let $\chi_{\min , \text { global }}^{2}$ be the $\chi^{2}$ at the global best fit point obtained from a combined fit to all the data. The quantity

$$
\chi_{\mathrm{PG}}^{2} \equiv \chi_{\min , \text { global }}^{2}-\chi_{\min , A}^{2}-\chi_{\min , B}^{2}=\Delta \chi_{A}^{2}+\Delta \chi_{B}^{2}
$$

measures by how much the fit worsens when the two data sets are combined. This can be seen from the second equality in eq. (A.1), in which we have defined, for each subset of the data, the $\chi^{2}$ difference $\Delta \chi_{A, B}^{2}$ between the individual best fit point and the global best fit point. If $\chi_{A}^{2}$ and $\chi_{B}^{2}$ depend on $P_{A}$ and $P_{B}$ parameters, respectively, and $P$ is the total number of parameters of the model $\left(P_{A}, P_{B} \leq P\right)$, then one can show [92] that $\chi_{\mathrm{PG}}^{2}$ follows a $\chi^{2}$ distribution with

$$
N_{\mathrm{PG}} \equiv P_{A}+P_{B}-P
$$

degrees of freedom. ${ }^{5}$ We can thus compute a $p$-value measuring the compatibility of the data sets $A$ and $B$ according to

$$
p=\int_{\chi_{\mathrm{PG}}^{2}}^{\infty} d x f_{\chi^{2}}\left(x ; N_{\mathrm{PG}}\right),
$$

where $f_{\chi^{2}}\left(x ; N_{\mathrm{PG}}\right)$ is the probability density function of the $\chi^{2}$ distribution with $N_{\mathrm{PG}}$ degrees of freedom.

\section{B Details of the IceCube fit}

The event numbers measured by the IceCube detector have been provided in a grid with 210 bins $[52,53]$, which depends on the reconstructed muon energy $E_{\mu}$ (logarithmically spaced in 10 bins ranging from $400 \mathrm{GeV}$ to $20 \mathrm{TeV}$ ) and the reconstructed muon direction (linearly spaced in 21 bins from $\cos \theta=-1.02$ to $\cos \theta=0.24$ ). We make the assumption

\footnotetext{
${ }^{5} N_{\mathrm{PG}}$ counts the number of "joint" parameters of the data sets $A$ and $B$. As an example, if $A$ and $B$ depend on exactly the same $P$ parameters, then $P_{A}=P_{B}=N_{\mathrm{PG}}=P$.
} 
that the reconstructed muon direction is the same as the direction of the initial neutrino. The predicted number of events in bin number $(i j)$ (where $i$ indexes $\cos \theta$ and $j$ indexes $\left.E_{\mu}\right)$ is computed according to

$$
\begin{aligned}
N_{i j}^{d, f}= & \int d E_{\nu}\left[\phi_{+}^{\mathrm{atm}, f}\left(E_{\nu}, \theta^{i}, N_{0}, \gamma, R_{\pi / K}\right) \bar{P}_{\mu \mu}^{+}\left(E_{\nu}, \theta^{i}\right) A_{\mathrm{eff},+}^{d}\left(E_{\nu}, E_{\mu}^{j}, \theta^{i}\right)\right. \\
& \left.+R_{ \pm} \phi_{-}^{\mathrm{atm}, f}\left(E_{\nu}, \theta^{i}, N_{0}, \gamma, R_{\pi / K}\right) \bar{P}_{\mu \mu}^{-}\left(E_{\nu}, \theta^{i}\right) A_{\mathrm{eff},-}^{d}\left(E_{\nu}, E_{\mu}^{j}, \theta^{i}\right)\right] .
\end{aligned}
$$

Here, $\phi_{ \pm}^{\text {atm }, f}\left(E_{\nu}, \theta^{i}, N_{0}, \gamma, R_{\pi / K}\right)$ is the atmospheric muon neutrino $(+)$ or anti-neutrino (-) flux, which depends on the true neutrino energy $E_{\nu}$, the neutrino direction $\theta^{i}$, and on the nuisance parameters $N_{0}, \gamma$, and $R_{\pi / K}$ discussed below. It also depends on the theoretical flux model, indicated by the subscript $f$. The effective area $A_{\text {eff, }}^{d}\left(E_{\nu}, E_{\mu}^{j}, \theta^{i}\right)$ in eq. (B.1) encodes the detector response to a $\nu_{\mu}(+)$ or $\bar{\nu}_{\mu}(-)$ with energy $E_{\nu}$ and direction $\theta^{i}$. The IceCube collaboration provides $A_{\text {eff, }}^{d}\left(E_{\nu}, E_{\mu}^{j}, \theta^{i}\right)$ in the form of a three-dimensional array in $E_{\mu}, \cos \theta$ (same binning as for the data), and $E_{\nu}$ (200 bins logarithmically spaced between $200 \mathrm{GeV}$ and $1 \mathrm{PeV}$ ) [52]. Separate arrays are provided for different assumptions on the Digital Optical Module (DOM) efficiency, indicated by the superscript $d$.

The muon neutrino and anti-neutrino survival probability $\bar{P}_{\mu \mu}^{ \pm}$is computed using GLoBES [126, 127], including a low-pass filter to suppress fast oscillation and to account for the limited energy resolution of the detector. For the production height of the neutrinos we interpolate linearly between $28 \mathrm{~km}$ for horizontal neutrinos and $18 \mathrm{~km}$ for vertical neutrinos [51]. To model the attenuation of the neutrino flux due to absorption in the Earth, we multiply the oscillation probability by an exponential damping factor given by

$$
e^{-X(\theta) \sigma^{ \pm}(E)\left(1-P_{\mu \mu}^{ \pm}\right)}
$$

where $X(\theta)$ is the column density along the neutrino trajectory and $\sigma^{ \pm}(E)$ the inclusive absorption cross-section for neutrinos and antineutrinos, respectively. The factor $\left(1-P_{\mu \mu}^{ \pm}(E, L)\right)$ accounts for the fact that only the active flavors interact with matter. This formula holds exactly only for an oscillation probability independent of the length of the trajectory. We make the assumption that in much of the parameter space the oscillations are either averaged out, or the oscillation length is so long that the probability is approximately constant along the trajectory. We have checked that our results do not depend significantly on this assumption.

In the published IceCube fit [52], systematic uncertainties are included either as discrete or as continuous nuisance parameters. The only discrete nuisance parameter in our analysis is the theoretical flux model. We found that out of the seven flux models considered by the IceCube collaboration, only two contribute significantly, namely the ones tagged "PolyGonato QGSJET-II-04" and "Honda-Gaisser". We therefore restrict our analysis to these two discrete models. Hence the index $f$ in eq. (B.1) runs from 1 to 2 .

The continuous nuisance parameters can be divided into two classes: those related to the neutrino flux, and those related to the detector response and the optical properties of the ice. In our analysis we use the following atmospheric neutrino flux uncertainties: 
- the normalization $N_{0}$. Formally we assume a large uncertainty of $40 \%$ on the normalization, but results are very similar for completely free normalization. Therefore we consider $N_{0}$ to be effectively unconstrained.

- the tilt of the energy spectrum, which is parameterized by including a factor $\left(E / E_{0}\right)^{\gamma}$, with a 5\% error on the power law index $\gamma$ and a central value of $\gamma=0$;

- the ratio between the pion and the kaon decay contributions to the flux, $R_{\pi / K}$, with an error of $10 \%$;

- the ratio between the neutrino and the anti-neutrino fluxes, $R_{ \pm}$, with an error of $2.5 \%$.

Out of the uncertainties associated with the detector response and the ice properties, we only include the uncertainty on the DOM efficiency. As stated above, the tabulated effective area is provided for four different models for the DOM efficiency. We interpolate linearly between the per-bin-prediction for each DOM model and allow the minimizer to choose the optimal superposition of DOM models. Concerning the ice properties, we restricted ourselves to the nominal model because effective areas for each DOM efficiencies are only provided for the nominal ice model.

For each point in the parameter space a $\chi^{2}$ value is calculated from the theoretical predictions and the experimental values by means of a log-likelihood function.

We have cross-check our IceCube fit with a second version of the analysis, which was developed completely independently. This analysis is not using the GLoBES software but is based on a dedicated probability code and it uses a partially different approach to systematics. The most noteworthy difference is the treatment of the discrete systematics. In our second implementation we restrict ourselves to only one flux model, the "HondaGaisser-model". Several other discrete systematics associated with the detector response are treated as continuous quantities, and their effects on the number of events are assumed to be linear. In detail, in our second implementation we use:

- the DOM efficiency, where as nominal value we have used the table corresponding to $99 \%$ efficiency, and as $1 \sigma$ deviation we have used the table corresponding to $95 \%$ efficiency;

- photon scattering in the ice, where the $1 \sigma$ deviation is defined from the table corresponding to a $10 \%$ increase with respect to the nominal response;

- photon absorption in the ice, where the $1 \sigma$ deviation is defined as a $10 \%$ increase in the absorption rate with respect to the nominal response;

- the azimuthal anisotropy in the scattering length due to the dust grain shear; here the $1 \sigma$ deviation is obtained from the data set denoted 'SPICELEA ice model';

- the optical properties of the ice column surrounding each string, where the $1 \sigma$ deviation is obtained from the data set labelled 'SPICEMIE ice model'. This data set does not include hole ice effects. 
Furthermore, in our second implementation, we average the oscillation probability over the altitude of the neutrino production point. The averaged probability is given by

$$
\left\langle P_{\mu \mu}^{ \pm}\left(E_{\nu}, \theta\right)\right\rangle=\int d h P_{\mu \mu}^{ \pm}\left(E_{\nu}, \cos \theta, h\right) \kappa^{ \pm}\left(E_{\nu}, \cos \theta, h\right),
$$

where $P_{\mu \mu}^{ \pm}\left(E_{\nu}, \cos \theta, h\right)$ is the unaveraged oscillation probability for a neutrino produced at altitude $h$ and $\kappa^{ \pm}\left(E_{\nu}, \cos \theta, h\right)$ is the distribution of production altitudes, normalized to one [51].

We find good agreement between our two implementations, and between each of our implementations and the official IceCube results [52]. We therefore conclude that our IceCube analysis is robust.

Open Access. This article is distributed under the terms of the Creative Commons Attribution License (CC-BY 4.0), which permits any use, distribution and reproduction in any medium, provided the original author(s) and source are credited.

\section{References}

[1] LSND collaboration, A.A. Aguilar-Arevalo et al., Evidence for neutrino oscillations from the observation of $\bar{\nu}_{e}$ appearance in a $\bar{\nu}_{\mu}$ beam, Phys. Rev. D 64 (2001) 112007 [hep-ex/0104049] [INSPIRE].

[2] MiniBoonE collaboration, A.A. Aguilar-Arevalo et al., Improved Search for $\bar{\nu}_{\mu} \rightarrow \bar{\nu}_{e}$ Oscillations in the MiniBooNE Experiment, Phys. Rev. Lett. 110 (2013) 161801 [arXiv: 1303.2588] [INSPIRE].

[3] T.A. Mueller et al., Improved Predictions of Reactor Antineutrino Spectra, Phys. Rev. C 83 (2011) 054615 [arXiv:1101.2663] [INSPIRE].

[4] P. Huber, On the determination of anti-neutrino spectra from nuclear reactors, Phys. Rev. C 84 (2011) 024617 [Erratum ibid. C 85 (2012) 029901] [arXiv:1106. 0687] [INSPIRE].

[5] G. Mention et al., The Reactor Antineutrino Anomaly, Phys. Rev. D 83 (2011) 073006 [arXiv: 1101.2755] [INSPIRE].

[6] A.C. Hayes, J.L. Friar, G.T. Garvey, G. Jungman and G. Jonkmans, Systematic Uncertainties in the Analysis of the Reactor Neutrino Anomaly, Phys. Rev. Lett. 112 (2014) 202501 [arXiv:1309.4146] [InSPIRE].

[7] D.-L. Fang and B.A. Brown, Effect of first forbidden decays on the shape of neutrino spectra, Phys. Rev. C 91 (2015) 025503 [Erratum ibid. C 93 (2016) 049903] [arXiv: 1502.02246] [INSPIRE].

[8] A.C. Hayes and P. Vogel, Reactor Neutrino Spectra, Ann. Rev. Nucl. Part. Sci. 66 (2016) 219 [arXiv: 1605.02047 ] [INSPIRE].

[9] M.A. Acero, C. Giunti and M. Laveder, Limits on $\nu_{e}$ and $\bar{\nu}_{e}$ disappearance from Gallium and reactor experiments, Phys. Rev. D 78 (2008) 073009 [arXiv:0711.4222] [INSPIRE].

[10] C. Giunti and M. Laveder, Statistical Significance of the Gallium Anomaly, Phys. Rev. C 83 (2011) 065504 [arXiv: 1006.3244] [INSPIRE].

[11] J. Kopp, M. Maltoni and T. Schwetz, Are there sterile neutrinos at the eV scale?, Phys. Rev. Lett. 107 (2011) 091801 [arXiv: 1103.4570] [INSPIRE]. 
[12] J.M. Conrad, C.M. Ignarra, G. Karagiorgi, M.H. Shaevitz and J. Spitz, Sterile Neutrino Fits to Short Baseline Neutrino Oscillation Measurements, Adv. High Energy Phys. 2013 (2013) 163897 [arXiv:1207.4765] [INSPIRE].

[13] M. Archidiacono, N. Fornengo, C. Giunti, S. Hannestad and A. Melchiorri, Sterile neutrinos: Cosmology versus short-baseline experiments, Phys. Rev. D 87 (2013) 125034 [arXiv:1302.6720] [INSPIRE].

[14] J. Kopp, P.A.N. Machado, M. Maltoni and T. Schwetz, Sterile Neutrino Oscillations: The Global Picture, JHEP 05 (2013) 050 [arXiv: 1303.3011] [INSPIRE].

[15] A. Mirizzi et al., The strongest bounds on active-sterile neutrino mixing after Planck data, Phys. Lett. B 726 (2013) 8 [arXiv:1303.5368] [InSPIRE].

[16] C. Giunti, M. Laveder, Y.F. Li and H.W. Long, Pragmatic View of Short-Baseline Neutrino Oscillations, Phys. Rev. D 88 (2013) 073008 [arXiv: 1308.5288] [INSPIRE].

[17] S. Gariazzo, C. Giunti and M. Laveder, Light Sterile Neutrinos in Cosmology and Short-Baseline Oscillation Experiments, JHEP 11 (2013) 211 [arXiv:1309.3192] [INSPIRE].

[18] G.H. Collin, C.A. Argüelles, J.M. Conrad and M.H. Shaevitz, Sterile Neutrino Fits to Short Baseline Data, Nucl. Phys. B 908 (2016) 354 [arXiv:1602.00671] [InSPIRE].

[19] S. Gariazzo, C. Giunti, M. Laveder and Y.F. Li, Updated Global $3+1$ Analysis of Short-BaseLine Neutrino Oscillations, JHEP 06 (2017) 135 [arXiv:1703.00860] [INSPIRE].

[20] C. Giunti, X.P. Ji, M. Laveder, Y.F. Li and B.R. Littlejohn, Reactor Fuel Fraction Information on the Antineutrino Anomaly, JHEP 10 (2017) 143 [arXiv:1708.01133] [INSPIRE].

[21] M. Dentler, Á. Hernández-Cabezudo, J. Kopp, M. Maltoni and T. Schwetz, Sterile neutrinos or flux uncertainties? - Status of the reactor anti-neutrino anomaly, JHEP 11 (2017) 099 [arXiv: 1709. 04294] [InSPIRE].

[22] Daya BAy collaboration, F.P. An et al., Improved Search for a Light Sterile Neutrino with the Full Configuration of the Daya Bay Experiment, Phys. Rev. Lett. 117 (2016) 151802 [arXiv: 1607.01174] [INSPIRE].

[23] NEOS collaboration, Y.J. Ko et al., Sterile Neutrino Search at the NEOS Experiment, Phys. Rev. Lett. 118 (2017) 121802 [arXiv:1610.05134] [INSPIRE].

[24] I. Alekseev et al., DANSS: Detector of the reactor AntiNeutrino based on Solid Scintillator, 2016 JINST 11 P11011 [arXiv: 1606.02896] [INSPIRE].

[25] M. Danilov, Search for sterile neutrinos at the DANSS and Neutrino-4 experiments talk given on behalf of the DANSS collaboration at the 52nd Rencontres de Moriond EW 2017, La Thuile, Italy, 18-25 March 2017 https://indico.in2p3.fr/event/13763/.

[26] M. Danilov, Search for sterile neutrinos at the DANSS experiment, talk given on behalf of the DANSS collaboration at the Solvay Workshop 'Beyond the Standard model with Neutrinos and Nuclear Physics', Brussels, Belgium, 29 November-1 December 2017.

[27] RENO collaboration, S.H. Seo et al., Spectral Measurement of the Electron Antineutrino Oscillation Amplitude and Frequency using 500 Live Days of RENO Data, Phys. Rev. D 98 (2018) 012002 [arXiv: 1610.04326] [INSPIRE].

[28] Double CHOOZ collaboration, Y. Abe et al., Improved measurements of the neutrino mixing angle $\theta_{13}$ with the Double CHOOZ detector, JHEP 10 (2014) 086 [Erratum JHEP 02 (2015) 074] [arXiv:1406.7763] [INSPIRE]. 
[29] DaYa Bay collaboration, F.P. An et al., Improved Measurement of the Reactor Antineutrino Flux and Spectrum at Daya Bay, Chin. Phys. C 41 (2017) 013002 [arXiv: 1607.05378] [INSPIRE].

[30] D.A. Dwyer and T.J. Langford, Spectral Structure of Electron Antineutrinos from Nuclear Reactors, Phys. Rev. Lett. 114 (2015) 012502 [arXiv: 1407.1281] [INSPIRE].

[31] A.C. Hayes et al., Possible origins and implications of the shoulder in reactor neutrino spectra, Phys. Rev. D 92 (2015) 033015 [arXiv:1506.00583] [InSPIRE].

[32] P. Novella, The antineutrino energy structure in reactor experiments, Adv. High Energy Phys. 2015 (2015) 364392 [arXiv:1512.03366] [INSPIRE].

[33] C. Giunti, Precise determination of the ${ }^{235} U$ reactor antineutrino cross section per fission, Phys. Lett. B 764 (2017) 145 [arXiv: 1608. 04096] [InSPIRE].

[34] P. Huber, NEOS Data and the Origin of the $5 \mathrm{MeV}$ Bump in the Reactor Antineutrino Spectrum, Phys. Rev. Lett. 118 (2017) 042502 [arXiv: 1609.03910] [INSPIRE].

[35] A.K. Mohanty, Possible origin of shoulder in the reactor antineutrino spectrum, arXiv: 1711.02801 [INSPIRE].

[36] J.M. Berryman, V. Brdar and P. Huber, Nuclear and Particle Conspiracy Solves Both Reactor Antineutrino Anomalies, arXiv:1803.08506 [INSPIRE].

[37] Daya Bay collaboration, F.P. An et al., Evolution of the Reactor Antineutrino Flux and Spectrum at Daya Bay, Phys. Rev. Lett. 118 (2017) 251801 [arXiv:1704.01082] [INSPIRE].

[38] P. Huber and P. Jaffke, Neutron capture and the antineutrino yield from nuclear reactors, Phys. Rev. Lett. 116 (2016) 122503 [arXiv:1510.08948] [INSPIRE].

[39] P. Huber, Nuclear physics and the reactor anomaly, talk given at the CERN Neutrino Platform Week, Center for Neutrino Physics at Virginia Tech, Blacksburg, Virginia, U.S.A., 29 January-2 February 2018 and online pdf version at https://indico.cern.ch/event/645835/ contributions/2777775/attachments/1593138/2522053/cern2018.pdf.

[40] OPERA collaboration, N. Agafonova et al., Search for $\nu_{\mu} \rightarrow \nu_{e}$ oscillations with the OPERA experiment in the CNGS beam, JHEP 07 (2013) 004 [Addendum JHEP 07 (2013) 085 ] [arXiv: 1303.3953] [INSPIRE].

[41] M. Antonello et al., Experimental search for the "LSND anomaly" with the ICARUS detector in the CNGS neutrino beam, Eur. Phys. J. C 73 (2013) 2345 [arXiv:1209.0122] [INSPIRE].

[42] C. Farnese, Results from ICARUS, talk given at the Neutrino 2014, Boston, Massachusetts, U.S.A., 2-7 June 2014 and online pdf version at https://indico.fnal.gov/event/8022/session/ 18/contribution/265/material/slides/0.pdf.

[43] MINOS collaboration, P. Adamson et al., Search for sterile neutrinos in MINOS and MINOS+ using a two-detector fit, submitted to Phys. Rev. Lett. (2017), arXiv: 1710.06488 [INSPIRE].

[44] NOvA collaboration, P. Adamson et al., Search for active-sterile neutrino mixing using neutral-current interactions in NOvA, Phys. Rev. D 96 (2017) 072006 [arXiv:1706. 04592] [INSPIRE].

[45] Y. Nakano, ${ }^{8}$ B solar neutrino spectrum measurement using Super-Kamiokande IV, Ph.D. Thesis, Tokyo University, Tokyo Japan (2016). 
[46] Borexino collaboration, G. Bellini et al., Neutrinos from the primary proton-proton fusion process in the Sun, Nature $\mathbf{5 1 2}$ (2014) 383 [INSPIRE].

[47] N. Vinyoles et al., A new Generation of Standard Solar Models, Astrophys. J. 835 (2017) 202 [arXiv: 1611.09867] [INSPIRE].

[48] Super-Kamiokande collaboration, R. Wendell, Atmospheric Results from Super-Kamiokande, AIP Conf. Proc. 1666 (2015) 100001 [arXiv:1412.5234] [InSPIRE] and online at https://indico.fnal.gov/event/8022/other-view?view=standard.

[49] ICECube collaboration, M.G. Aartsen et al., Determining neutrino oscillation parameters from atmospheric muon neutrino disappearance with three years of IceCube DeepCore data, Phys. Rev. D 91 (2015) 072004 [arXiv:1410.7227] [INSPIRE].

[50] IceCube collaboration, J.P. Yañez et al., IceCube Oscillations: 3 years muon neutrino disappearance data, (2015) https://icecube.wisc.edu/science/data/nu_osc.

[51] M. Honda, M. Sajjad Athar, T. Kajita, K. Kasahara and S. Midorikawa, Atmospheric neutrino flux calculation using the NRLMSISE-00 atmospheric model, Phys. Rev. D 92 (2015) 023004 [arXiv:1502.03916] [INSPIRE].

[52] IceCube collaboration, M.G. Aartsen et al., Searches for Sterile Neutrinos with the IceCube Detector, Phys. Rev. Lett. 117 (2016) 071801 [arXiv: 1605.01990] [INSPIRE] and online at http://icecube.wisc.edu/science/data/IC86-sterile-neutrino.

[53] B.J.P. Jones, Sterile neutrinos in cold climates, Ph.D. Thesis, Massachusetts Institute of Technology, Cambridge Massachusetts U.S.A. (2015) and online at http://hdl.handle.net/1721.1/101327.

[54] C.A. Argüelles, New Physics with Atmospheric Neutrinos, Ph.D. Thesis, University of Wisconsin, Madison Wisconsin U.S.A. (2015) and online pdf version at https://docushare.icecube.wisc.edu/dsweb/Get/Document-75669/tesis.pdf.

[55] L. Wolfenstein, Neutrino Oscillations in Matter, Phys. Rev. D 17 (1978) 2369 [InSPIRE].

[56] S.P. Mikheyev and A.Y. Smirnov, Resonance Amplification of Oscillations in Matter and Spectroscopy of Solar Neutrinos, Sov. J. Nucl. Phys. 42 (1985) 913 [Yad. Fiz. 42 (1985) 1441] [INSPIRE].

[57] H. Nunokawa, O.L.G. Peres and R. Zukanovich Funchal, Probing the LSND mass scale and four neutrino scenarios with a neutrino telescope, Phys. Lett. B 562 (2003) 279 [hep-ph/0302039] [INSPIRE].

[58] S. Choubey, Signature of sterile species in atmospheric neutrino data at neutrino telescopes, JHEP 12 (2007) 014 [arXiv:0709.1937] [INSPIRE].

[59] H. Kwon et al., Search for Neutrino Oscillations at a Fission Reactor, Phys. Rev. D 24 (1981) 1097 [INSPIRE].

[60] CALTECH-SIN-TUM collaboration, G. Zacek et al., Neutrino Oscillation Experiments at the Gosgen Nuclear Power Reactor, Phys. Rev. D 34 (1986) 2621 [INSPIRE].

[61] G.S. Vidyakin et al., Detection of Anti-neutrinos in the Flux From Two Reactors, Sov. Phys. JETP 66 (1987) 243 [Zh. Eksp. Teor. Fiz. 93 (1987) 424] [INSPIRE].

[62] G.S. Vidyakin et al., Limitations on the characteristics of neutrino oscillations, JETP Lett. 59 (1994) 390 [Pisma Zh. Eksp. Teor. Fiz. 59 (1994) 364] [INSPIRE].

[63] Y.V. Kozlov et al., Today and future neutrino experiments at Krasnoyarsk nuclear reactor, Nucl. Phys. Proc. Suppl. 87 (2000) 514 [hep-ex/9912046] [inSPIRE]. 
[64] A.I. Afonin, S.N. Ketov, V.I. Kopeikin, L.A. Mikaelyan, M.D. Skorokhvatov and S.V. Tolokonnikov, A study of the reaction $\bar{\nu}_{e}+p \rightarrow e^{+}+n$ on a nuclear reactor, Sov. Phys. JETP 67 (1988) 213 [INSPIRE].

[65] A.A. Kuvshinnikov, L.A. Mikaelyan, S.V. Nikolaev, M.D. Skorokhvatov and A.V. Etenko, Measuring the $\bar{\nu}_{e}+p \rightarrow n+e^{+}$cross-section and beta decay axial constant in a new experiment at Rovno NPP reactor (in Russian), JETP Lett. 54 (1991) 253 [INSPIRE].

[66] Y. Declais et al., Search for neutrino oscillations at 15-meters, 40-meters and 95-meters from a nuclear power reactor at Bugey, Nucl. Phys. B 434 (1995) 503 [INSPIRE].

[67] Y. Declais et al., Study of reactor anti-neutrino interaction with proton at Bugey nuclear power plant, Phys. Lett. B 338 (1994) 383 [INSPIRE].

[68] Z.D. Greenwood et al., Results of a two position reactor neutrino oscillation experiment, Phys. Rev. D 53 (1996) 6054 [InSPIRE].

[69] H. Seo, New Results from RENO, talk given on behalf of the RENO collaboration at the EPS conference on High Energy Physics, Venice, Italy, 5-11 July 2017.

[70] S.-H. Seo, New Results from RENO, talk given on behalf of the RENO collaboration at the XXVI International Conference on Neutrino Physics and Astrophysics, Boston, Massachusetts, U.S.A., 2-7 June 2014.

[71] Daya Bay collaboration, F.P. An et al., Measurement of electron antineutrino oscillation based on 1230 days of operation of the Daya Bay experiment, Phys. Rev. D 95 (2017) 072006 [arXiv: 1610.04802] [INSPIRE].

[72] KamLAND collaboration, A. Gando et al., Constraints on $\theta_{13}$ from A Three-Flavor Oscillation Analysis of Reactor Antineutrinos at KamLAND, Phys. Rev. D 83 (2011) 052002 [arXiv: 1009.4771] [INSPIRE].

[73] B.T. Cleveland et al., Measurement of the solar electron neutrino flux with the Homestake chlorine detector, Astrophys. J. 496 (1998) 505 [INSPIRE].

[74] F. Kaether, W. Hampel, G. Heusser, J. Kiko and T. Kirsten, Reanalysis of the GALLEX solar neutrino flux and source experiments, Phys. Lett. B 685 (2010) 47 [arXiv: 1001.2731] [INSPIRE].

[75] SAGE collaboration, J.N. Abdurashitov et al., Measurement of the solar neutrino capture rate with gallium metal. III: Results for the 2002-2007 data-taking period, Phys. Rev. C 80 (2009) 015807 [arXiv:0901.2200] [INSPIRE].

[76] Super-Kamiokande collaboration, J. Hosaka et al., Solar neutrino measurements in Super-Kamiokande-I, Phys. Rev. D 73 (2006) 112001 [hep-ex/0508053] [INSPIRE].

[77] Super-Kamiokande collaboration, J.P. Cravens et al., Solar neutrino measurements in Super-Kamiokande-II, Phys. Rev. D 78 (2008) 032002 [arXiv:0803.4312] [INSPIRE].

[78] Super-Kamiokande collaboration, K. Abe et al., Solar neutrino results in Super-Kamiokande-III, Phys. Rev. D 83 (2011) 052010 [arXiv: 1010.0118] [INSPIRE].

[79] SNO collaboration, B. Aharmim et al., Determination of the $\nu_{e}$ and total ${ }^{8} B$ solar neutrino fluxes with the Sudbury neutrino observatory phase I data set, Phys. Rev. C 75 (2007) 045502 [nucl-ex/0610020] [inSPIRE].

[80] SNO collaboration, B. Aharmim et al., Electron energy spectra, fluxes and day-night asymmetries of ${ }^{8} \mathrm{~B}$ solar neutrinos from measurements with $\mathrm{NaCl}$ dissolved in the heavy-water detector at the Sudbury Neutrino Observatory, Phys. Rev. C 72 (2005) 055502 [nucl-ex/0502021] [INSPIRE]. 
[81] SNO collaboration, B. Aharmim et al., An Independent Measurement of the Total Active ${ }^{8} B$ Solar Neutrino Flux Using an Array of ${ }^{3}$ He Proportional Counters at the Sudbury Neutrino Observatory, Phys. Rev. Lett. 101 (2008) 111301 [arXiv:0806.0989] [INSPIRE].

[82] Borexino collaboration, G. Bellini et al., Precision measurement of the ${ }^{7}$ Be solar neutrino interaction rate in Borexino, Phys. Rev. Lett. 107 (2011) 141302 [arXiv:1104.1816] [INSPIRE].

[83] Borexino collaboration, G. Bellini et al., Measurement of the solar ${ }^{8} B$ neutrino rate with a liquid scintillator target and $3 \mathrm{MeV}$ energy threshold in the Borexino detector, Phys. Rev. D 82 (2010) 033006 [arXiv:0808.2868] [INSPIRE].

[84] J. Reichenbacher, Final KARMEN results on neutrino oscillations and neutrino nucleus interactions in the energy regime of supernovae, Ph.D. Thesis, Karlsruhe Universität, Karlsruhe Germany (2005).

[85] B. Armbruster et al., KARMEN limits on $\nu_{e} \rightarrow \nu_{\tau}$ oscillations in two neutrino and three neutrino mixing schemes, Phys. Rev. C 57 (1998) 3414 [hep-ex/9801007] [INSPIRE].

[86] J.M. Conrad and M.H. Shaevitz, Limits on Electron Neutrino Disappearance from the KARMEN and LSND $\nu_{e}$-Carbon Cross Section Data, Phys. Rev. D 85 (2012) 013017 [arXiv: 1106.5552] [INSPIRE].

[87] LSND collaboration, L.B. Auerbach et al., Measurements of charged current reactions of $\nu_{e}$ on ${ }^{12}$ C, Phys. Rev. C 64 (2001) 065501 [hep-ex/0105068] [INSPIRE].

[88] GALLEX collaboration, W. Hampel et al., Final results of the ${ }^{51} \mathrm{Cr}$ neutrino source experiments in GALLEX, Phys. Lett. B 420 (1998) 114 [INSPIRE].

[89] SAGE collaboration, J.N. Abdurashitov et al., Measurement of the response of the Russian-American gallium experiment to neutrinos from a ${ }^{51} \mathrm{Cr}$ source, Phys. Rev. C 59 (1999) 2246 [hep-ph/9803418] [INSPIRE].

[90] J.N. Abdurashitov et al., Measurement of the response of a Ga solar neutrino experiment to neutrinos from an ${ }^{37}$ Ar source, Phys. Rev. C 73 (2006) 045805 [nucl-ex/0512041] [INSPIRE].

[91] S. Gariazzo, C. Giunti, M. Laveder and Y.F. Li, Model-independent $\bar{\nu}_{e}$ short-baseline oscillations from reactor spectral ratios, Phys. Lett. B 782 (2018) 13 [arXiv:1801.06467] [INSPIRE].

[92] M. Maltoni and T. Schwetz, Testing the statistical compatibility of independent data sets, Phys. Rev. D 68 (2003) 033020 [hep-ph/0304176] [INSPIRE].

[93] M. Maltoni and T. Schwetz, Sterile neutrino oscillations after first MiniBooNE results, Phys. Rev. D 76 (2007) 093005 [arXiv:0705.0107] [inSPIRE].

[94] KARMEN collaboration, B. Armbruster et al., Upper limits for neutrino oscillations $\bar{\nu}_{\mu} \rightarrow \bar{\nu}_{e}$ from muon decay at rest, Phys. Rev. D 65 (2002) 112001 [hep-ex/0203021] [INSPIRE].

[95] NOMAD collaboration, P. Astier et al., Search for $\nu_{\mu} \rightarrow \nu_{e}$ oscillations in the NOMAD experiment, Phys. Lett. B 570 (2003) 19 [hep-ex/0306037] [INSPIRE].

[96] L. Borodovsky et al., Search for muon-neutrino oscillations $\nu_{\mu} \rightarrow \nu_{e}\left(\bar{\nu}_{\mu} \rightarrow \bar{\nu}_{e}\right)$ in a wide band neutrino beam, Phys. Rev. Lett. 68 (1992) 274 [INSPIRE].

[97] C. Farnese, Some recent results from ICARUS, AIP Conf. Proc. 1666 (2015) 110002 [INSPIRE] and online at https://indico.fnal.gov/materialDisplay.py?contribId= $265 \&$ sessionId $=18 \&$ materialId $=$ slides\&confId $=8022$. 
[98] MiniBoone collaboration, A.A. Aguilar-Arevalo et al., Data release for arXiv:1207.4809, (2012) and online at http://www-boone.fnal.gov/for_physicists/data_release/ nue_nuebar_2012/combined.html\#fit200.

[99] M. Maltoni, T. Schwetz, M.A. Tortola and J.W.F. Valle, Ruling out four neutrino oscillation interpretations of the LSND anomaly?, Nucl. Phys. B 643 (2002) 321 [hep-ph/0207157] [INSPIRE].

[100] F. Dydak et al., A Search for $\nu_{\mu}$ oscillations in the $\Delta \mathrm{m}^{2}$ range $0.3-90 \mathrm{eV}^{2}$, Phys. Lett. B 134 (1984) 281 [inSPIRE].

[101] MiniBoonE collaboration, A.A. Aguilar-Arevalo et al., A Search for muon neutrino and antineutrino disappearance in MiniBooNE, Phys. Rev. Lett. 103 (2009) 061802 [arXiv:0903.2465] [INSPIRE].

[102] SciBoonE and MiniBoone collaborations, G. Cheng et al., Dual baseline search for muon antineutrino disappearance at $0.1 \mathrm{eV}^{2}<\Delta m^{2}<100 \mathrm{eV}^{2}$, Phys. Rev. D 86 (2012) 052009 [arXiv: 1208.0322] [INSPIRE].

[103] Super-Kamiokande collaboration, R. Wendell et al., Atmospheric neutrino oscillation analysis with sub-leading effects in Super-Kamiokande I, II and III, Phys. Rev. D 81 (2010) 092004 [arXiv: 1002 . 3471] [INSPIRE].

[104] MINOS collaboration, P. Adamson et al., Search for Sterile Neutrinos Mixing with Muon Neutrinos in MINOS, Phys. Rev. Lett. 117 (2016) 151803 [arXiv:1607.01176] [INSPIRE].

[105] A.M. Dziewonski and D.L. Anderson, Preliminary Reference Earth Model, Phys. Earth Planet. Interiors 25 (1981) 297 [INSPIRE].

[106] W.C. Louis, Problems With the MINOS/MINOS+ Sterile Neutrino $\nu_{\mu}$ Result, arXiv: 1803.11488 [INSPIRE].

[107] MiniBooNE collaboration, A.A. Aguilar-Arevalo et al., Data release for arXiv:0903.2465, (2009) and online at http://www-boone.fnal.gov/for_physicists/data_release/ numu_numubar/.

[108] M. Blennow, E. Fernandez-Martinez, J. Gehrlein, J. Hernandez-Garcia and J. Salvado, IceCube bounds on sterile neutrinos above $10 \mathrm{eV}$, arXiv:1803.02362 [INSPIRE].

[109] S.M. Bilenky, C. Giunti and W. Grimus, Neutrino mass spectrum from the results of neutrino oscillation experiments, Eur. Phys. J. C 1 (1998) 247 [hep-ph/9607372] [INSPIRE].

[110] N. Okada and O. Yasuda, A Sterile neutrino scenario constrained by experiments and cosmology, Int. J. Mod. Phys. A 12 (1997) 3669 [hep-ph/9606411] [INSPIRE].

[111] V.D. Barger, S. Pakvasa, T.J. Weiler and K. Whisnant, Variations on four neutrino oscillations, Phys. Rev. D 58 (1998) 093016 [hep-ph/9806328] [INSPIRE].

[112] R.H. Cyburt, B.D. Fields, K.A. Olive and T.-H. Yeh, Big Bang Nucleosynthesis: 2015, Rev. Mod. Phys. 88 (2016) 015004 [arXiv: 1505.01076] [INSPIRE].

[113] Planck collaboration, P.A.R. Ade et al., Planck 2015 results. XIII. Cosmological parameters, Astron. Astrophys. 594 (2016) A13 [arXiv:1502.01589] [INSPIRE].

[114] B. Dasgupta and J. Kopp, Cosmologically Safe eV-Scale Sterile Neutrinos and Improved Dark Matter Structure, Phys. Rev. Lett. 112 (2014) 031803 [arXiv:1310.6337] [INSPIRE].

[115] S. Hannestad, R.S. Hansen and T. Tram, How Self-Interactions can Reconcile Sterile Neutrinos with Cosmology, Phys. Rev. Lett. 112 (2014) 031802 [arXiv:1310.5926] [INSPIRE]. 
[116] X. Chu, B. Dasgupta, M. Dentler, J. Kopp and N. Saviano, Sterile Neutrinos with Secret Interactions - Cosmological Discord?, arXiv:1806.10629 [INSPIRE].

[117] J.F. Cherry, A. Friedland and I.M. Shoemaker, Short-baseline neutrino oscillations, Planck and IceCube, arXiv:1605.06506 [InSPIRE].

[118] C.E. Yaguna, Sterile neutrino production in models with low reheating temperatures, JHEP 06 (2007) 002 [arXiv:0706.0178] [INSPIRE].

[119] N. Saviano, A. Mirizzi, O. Pisanti, P.D. Serpico, G. Mangano and G. Miele, Multi-momentum and multi-flavour active-sterile neutrino oscillations in the early universe: role of neutrino asymmetries and effects on nucleosynthesis, Phys. Rev. D 87 (2013) 073006 [arXiv: 1302.1200] [INSPIRE].

[120] C.M. Ho and R.J. Scherrer, Sterile Neutrinos and Light Dark Matter Save Each Other, Phys. Rev. D 87 (2013) 065016 [arXiv: 1212.1689] [INSPIRE].

[121] M. Giovannini, H. Kurki-Suonio and E. Sihvola, Big bang nucleosynthesis, matter antimatter regions, extra relativistic species and relic gravitational waves, Phys. Rev. D 66 (2002) 043504 [astro-ph/0203430] [INSPIRE].

[122] J.L. Bernal, L. Verde and A.G. Riess, The trouble with $H_{0}$, JCAP 10 (2016) 019 [arXiv: 1607.05617 ] [INSPIRE].

[123] STEREO collaboration, H. Almazán et al., Sterile neutrino exclusion from the STEREO experiment with 66 days of reactor-on data, arXiv:1806.02096 [INSPIRE].

[124] PROSPECT collaboration, J. Ashenfelter et al., First search for short-baseline neutrino oscillations at HFIR with PROSPECT, arXiv:1806.02784 [INSPIRE].

[125] MiniBoonE collaboration, A.A. Aguilar-Arevalo et al., Observation of a Significant Excess of Electron-Like Events in the MiniBooNE Short-Baseline Neutrino Experiment, arXiv: 1805.12028 [INSPIRE].

[126] P. Huber, M. Lindner and W. Winter, Simulation of long-baseline neutrino oscillation experiments with GLoBES (General Long Baseline Experiment Simulator), Comput. Phys. Commun. 167 (2005) 195 [hep-ph/0407333] [INSPIRE].

[127] P. Huber, J. Kopp, M. Lindner, M. Rolinec and W. Winter, New features in the simulation of neutrino oscillation experiments with GLoBES 3.0: General Long Baseline Experiment Simulator, Comput. Phys. Commun. 177 (2007) 432 [hep-ph/0701187] [INSPIRE]. 